\title{
Article
}

\section{Dark Matter and Its Relationship with Gravity, Electromagnetism and the Two Nuclear Forces}

\author{
Engel Roza \\ Stripperwei 1, 5551 ST Valkenswaard, The Netherlands \\ Email: engel.roza@onsbrabantnet.nl
}

\begin{abstract}
Summary
In a search for a solution of the galaxy rotation problem, a novel view on cosmological gravity is developed. In this view, the origin of gravity is traced back to the basic nuclear field of energy, spread by quarks as elementary pointlike sources. This gives a common basis for gravity, electromagnetism, the strong nuclear force and the weak nuclear force. After a review on gravimagnetism and the causality problem of gravity, a hypothetical concept for the gravity field is proposed, which solves the rotation problem without the need to accept dark matter as the deus ex machina.
\end{abstract}

Keywords: dark matter; cosmological gravity; galaxy rotation problem; gravimagnetism; antigravity; cosmological constant

\section{Introduction}

Dark matter is a hypothetical physical massive substance, the existence of which is proposed to explain particular phenomena observed in cosmology that cannot be understood from common physical laws. An intriguing example of such a phenomenon is the experimental evidence that stars rotating at the edge of a galaxy show an orbital velocity that remains more or less constant as a function of their spacing from the galaxy center. Although this phenomenon can be explained for particular mass density distributions, the orbital velocity appears to be larger than allowed by the Newtonian law of gravity, which limits this velocity by the amount of enclosed mass. Therefore, "something else" should be responsible for the phenomenon of excessive orbital velocity.

Several mechanisms have been proposed to explain the unexpected. The hypothetical existence of mass within the galaxy that escapes from visible observation, is one of these. Such dark matter would explain an increase of the gravitational force, thereby making the orbital velocity higher than expected from the amount of visible mass. Another mechanism is the Milgrom's MOND hypothesis [1]. MOND stands for MOdified Newtonian Dynamics. In this approach, it is hypothesized that Newton's gravitational law is incomplete and that it can be adapted in a way that its influence becomes manifest at a cosmological scale and remains hidden in our daily world. This adaption, however, is not clearly physically justified, but is made on the basis of a curve fit to empirical results from observations. A third mechanism is proposed by Verlinde [2], who regards gravitation as an entropy phenomenon, emergent from other physical laws, rather than as a fundamental force of nature. He adopts the holographic principle of the string theory. According to this principle, the physical laws of our three-dimensional world, allows a (two-dimensional) holographic mapping on a shell around space. Verlinde hypothesizes fictitious molecules on this shell, which show the entropy as prescribed by the second law of thermodynamics. Physical mass objects in this fictitious world appear to show a motion behavior that can be described mathematically in a format that equals Newton's law of gravity. He claims that, similarly as with MOND, this mechanism gives a clue to understand the behavior of mass objects at a cosmological scale without the need to accept the existence of dark matter. 
In this article, I wish to show that there might be fundamental physical reasons why mass objects in deep space behave differently from the behavior of mass objects in noncosmological space. These reasons have to do with the relationship between mass, gravity, electromagnetism and the two basic nuclear forces. It all starts with the concept of quarks as described in previous work $[3,4]$. In this concept, a bare quark is a massless pointlike source of an energetic flux. This flux produces two energetic fields, namely a vector type repelling "far field" and a scalar type attracting "near field". This enables the origin of stable bonds between two quarks, known as mesons, and between three quarks, known as baryons. These configurations behave as quantum mechanical oscillators. The energetic state of these oscillators becomes manifest as mass. A quark on its own, has no relevant mass attribute. Mass is the consequence of the quantum mechanical junction of quarks (confinement). Electric charge is a consequence of this junction as well. It is the manifestation of the (iso)spin condition of the quark junction. As a result, electric charge is quantized in integer units. Mass is quantized as well, albeit as a quantization level of the quantum mechanical oscillator state. This shows an irregular energy spacing. It is due to the characteristics of this oscillator, which is not purely harmonic as it would be in a quadratic field of energy. Instead, it is slightly anharmonic as a consequence of the Proca type of the quark's nuclear far field and the Yukawa type of the nuclear near field. There is, however, a clear parallel. Electromagnetism, which originates from the phenomenon of electric charge, and gravity, which originates from the phenomenon of mass, are indirect forces that follow from the existence of the energetic field of the two basic nuclear forces.

We know that the electromagnetic force has a vector potential next to a scalar potential. Taking into account the parallel between electromagnetism and gravity as just described, it would be reasonable to suppose that such would hold for gravity as well. So, where is the vector potential of gravity? It might well be that this potential escapes from our observation because of its weakness. Would it be possible that this vector potential may show its existence at the cosmological scale? It is the aim of this article, to explore if such could be true. If gravity would be incomplete, in the sense that next to its scalar field a hidden vectorial field would exist, it could well be possible that currents of mass particles would show similar properties as currents of electrically charged particles. In that case, mass currents would create the equivalent of a magnetic field and so could execute the equivalent of the Lorentz force on other mass currents. Such an effect may manifest itself at a cosmological scale as the force that enhances the Newtonian force, thereby suggesting that some dark matter increases the strength of the scalar gravity force.

The idea of gravimagnetism is not new. In fact, it traces back to Heaviside [5]. The present approach to it is based upon a relativistic formalism, derived from Einstein's Field Equation. The suggestion that the (equivalent) Lorentz force might be related with dark matter is not new either. Attempts to explain the dark matter phenomenon in terms of a gravimagnetic Lorentz force have remained unsuccessfully, because its strength is considered as being too weak. In this article, I wish to review the dark matter problem once more as well as the shortcoming of gravimagnetism to explain outstanding cosmological problems. After that, I'Il propose a novel concept of Cosmological Gravity in an attempt to find a way out. 


\section{The galaxy rotation curve problem: dark matter or dark force?}

Newtonian laws prescribe that the transverse velocity $v_{\varphi}(r)$ of a cosmic object revolving in a circular orbit with radius $r$ in a gravity field is determined by

$v_{\varphi}^{2}(r)=\frac{M(r) G}{r}$.

where $M(r)$ is the amount of enclosed mass and where $G$ is the gravitational constant. This relationship is often denoted as Kepler's third law. As illustrated in figure 1, the velocity curve of cosmic objects in a galaxy, such as, for instance, the Milky Way, appears to be almost flat. It is tempting to believe that this can be due to a particular spectral distribution of the spectral density to compose $M(r)$. This, however, cannot be true, because $M(r)$ builds up to a constant value of the overall mass. And Kepler's law states in fact that a flat mass curve $M(r)$ is not compatible with a flat velocity curve.

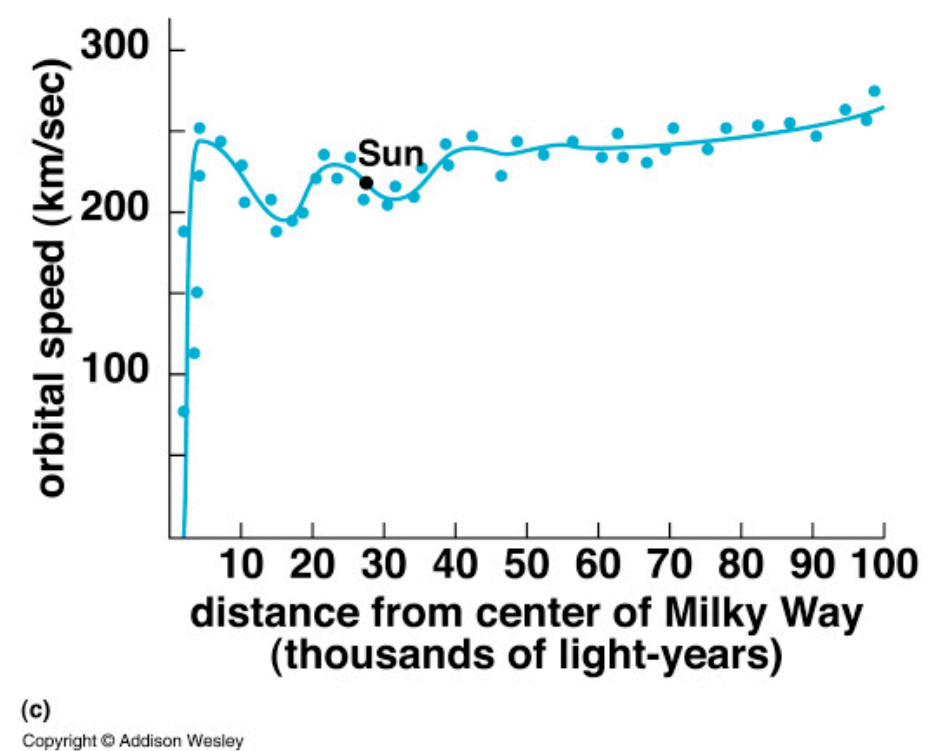

Figure 1: Rotation curves of solar objects in the Milky Way (From ircamera.as.arizona.edu)

Figure 2 illustrates the problem. It is one of the two: either some additional force on top of the Newtonian law is responsible for the phenomenon, or dark matter, affecting the mass distribution is responsible. Apart from this, it can be readily concluded from Table I that the mass data of the Milky Way are inadequate to explain the high value of $v_{\varphi}(r)$ at the edge of the galaxy. Figure 1 shows that the revolution speed of the sun (at $R=8 \mathrm{kpc}$ ) in the Milky Way has about the same value of some $220 \mathrm{~km} / \mathrm{s}$ as the one of a star at the edge of the Milky Way $(R=20 \mathrm{kpc}$ ). For a stellar object at the edge, all Milky Way mass can be considered as if it were central. Using the values shown in Table I, it would result into a revolution speed of $132 \mathrm{~km} / \mathrm{s}$. The amount of missing mass can thus be estimated from

$$
\frac{M_{G}+M_{\text {dark }}}{M_{G}}=\frac{1}{1-\alpha}=\left(\frac{220}{132}\right)^{2} \rightarrow \alpha=0.64
$$


It means that some $64 \%$ of the Milky Way would consist of dark matter.

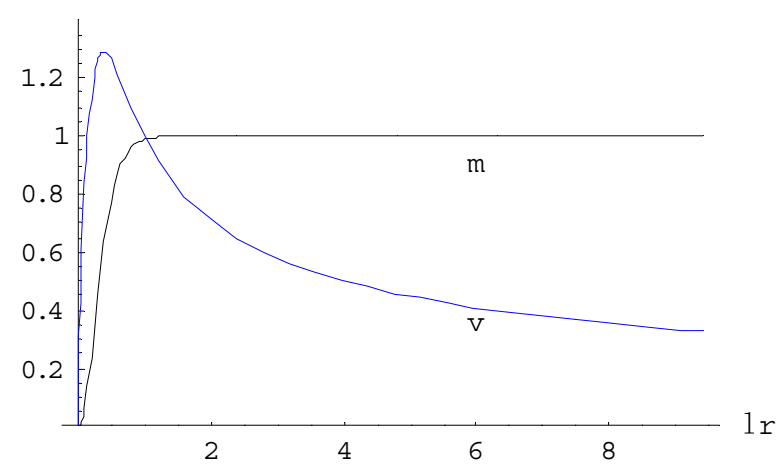

Figure 2. Incompatibility of a flat enclosed mass curve with a flat rotation curve.

Table I. Characteristics of the Milky Way

\begin{tabular}{|l|l|l|}
\hline distance unit & $1 \mathrm{kpc}$ & $3.0610^{19}[\mathrm{~m}]$ \\
\hline grav. constant & $G$ & $6.67 \times 10^{-11}\left[\mathrm{~m}^{3} \mathrm{~kg}^{-1} \mathrm{~s}^{-2}\right]$ \\
\hline solar mass & $M_{\text {sol }}$ & $1.9910^{30}[\mathrm{~kg}]$ \\
\hline mass of the bulge & $M_{b u \text { lge }}$ & $2 \times 10^{10} M_{\text {sol }}$ \\
\hline mass of the disc & $M_{\text {disc }}$ & $6 \times 10^{10} M_{\text {sol }}$ \\
\hline Mass Milky Way & $M_{R}$ & $8 \times 10^{10} M_{\text {sol }}$ \\
\hline Radius Milky Way & $R$ & $20 \mathrm{kpc}$ \\
\hline
\end{tabular}

\section{Gravimagnetism as dark force?}

As already stated, another possibility for explaining the anomalous rotation curve is the hypothetical existence of some dark force. Let us consider if the concept of gravimagnetism may reveal such. Many galaxies, such as, for instance, the Milky Way show a typical topological structure of the type as shown in figure 3.

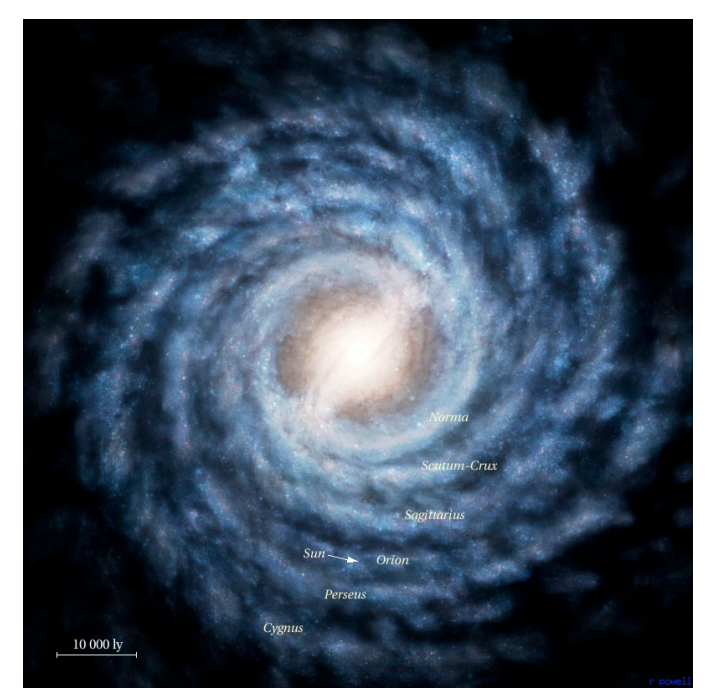

Figure 3: Milky Way (from http://www.atlasoftheuniverse.com/milkyway2.jpg) 
This structure is a collection of spiral arms in which solar objects show an orbital motion around the center of the galaxy. It will be clear that neighboring arms can be conceived as parallel currents of massive particles. In terms of the analogy between electromagnetism and gravity, such as summarized in Table II, one might hypothesize that these currents execute an attracting Lorentz force on each other of the type as shown in the last row of Table I. This would mean that, apart from the Newtonian gravity force that attracts massive objects to the center of the galaxy, an additional central force influences the equation of motion of such masses. This effect is formally expressed in the energy relationship

$$
\frac{1}{2} m v^{2}+\frac{1}{2} J \omega^{2}+V_{G}(r)+V_{L}(r)=E
$$

where $m$ is the mass of the particle, $v$ its radial velocity, $J$ its moment of inertia with respect to the galaxy center, $\omega(=\mathrm{d} \varphi / \mathrm{d} t)$ its angular frequency, $V_{G}$ its potential energy due to gravity, $V_{L}$ its potential energy due to the Lorentz force and $E$ the total particle's energy. Next to conservation of energy as expressed by (3), the angular momentum is a conserved quantity, so

$$
m r^{2} \frac{\mathrm{d} \varphi}{\mathrm{d} t}=b
$$

where $b$ is a constant. It is well known that, by evaluating of this expression for the case that $V_{G}$ is the potential energy associated with Newton's gravity law and that no Lorentz force is involved, under consideration of these conservation laws, elliptic orbits appear for the motion of the mass particle $m$. Among the variety of elliptic orbits possible, the circular motion, where the radial velocity $v=0$, is a valid special case. As shown in figure 3 , orbits in the Milky Way are more or less circular.

Table II: Analogy of (naive) gravimagnetism with electromagnetism.

\begin{tabular}{|l|l|l|l|}
\hline & electric & gravitational & (gravi)dimension \\
\hline current & $I=\Delta q / \Delta t$ & $I=\Delta m / \Delta t$ & {$\left[\mathrm{~kg} \mathrm{~s}^{-1}\right]$} \\
\hline Coulomb/Newton & $F=q_{1} q_{2} / 4 \pi \varepsilon_{0} r^{2}$ & $F=m_{1} m_{2} G / r^{2}$ & {$\left[\mathrm{~kg} \mathrm{~m} \mathrm{~s}^{-2}\right]$} \\
\hline Lorentz force & $q v \times B$ & $m v \times B$ & {$\left[\mathrm{~kg} \mathrm{~m} \mathrm{~s}^{-2}\right]$} \\
\hline (gravi) magn. field & $B=\mu_{0} I / 2 r$ & $B=\mu_{G} I / 2 r$ & {$\left[\mathrm{~s}^{-1}\right]$} \\
\hline constant of nature & $\varepsilon_{0}$ & $\varepsilon_{G}=1 / 4 \pi G$ & {$\left[\left(\mathrm{~m}^{3} \mathrm{~kg}^{-1} \mathrm{~s}^{-2}\right)^{-1}\right]$} \\
\hline propagation speed & $c=\left(\varepsilon_{0} \mu_{0}\right)^{-1 / 2}$ & $c$ & {$\left[\mathrm{~m} \mathrm{~s}^{-1}\right]$} \\
\hline gravimagnetic constant & & $\mu_{G}=\left(c_{G}^{2} \varepsilon_{G}\right)^{-1}$ & {$\left[\mathrm{~kg}^{-1} \mathrm{~m}\right]$} \\
\hline $\begin{array}{l}\text { force between two } \\
l \text { long current wires }\end{array}$ & $F=\mu_{0} I_{1} I_{2} l / 2 \pi r$ & $F=\mu_{G} I_{1} I_{2} l / 2 \pi r$ & {$\left[\mathrm{~kg} \mathrm{~m} \mathrm{~s}^{-2}\right]$} \\
\hline
\end{tabular}

This picture shows a core (the bulge) and a number of spiral arms. Both the bulge and the massive objects (stellar) in the spiral arms rotate around the center. Experimental evidence 
shows that the tangential velocity of the stellars is different from the Keplian law as expressed by (1). As discussed before and shown in figure 1, this value appears to approach a value independent of $r$, at remote distances from the center. A few number of spiral arms can be distinguished. These spiral arms have a certain width and a certain spacing between them. To keep the model simple, it will be supposed that the width $\Delta$ of the spiral arms is about the same as the spacing between them. The particle streams in the arms will be considered as gravitational currents $I_{i}$ in parallel wires. In accordance with the hypothetical equivalence of electromagnetism and gravity, the currents in the arms execute a gravitational Lorentz force that can be calculated according to the formula as shown in the last row of Table I. This builds a certain potential energy $U_{L}(r)$ of the outer spiral arm with respect to the galaxy center, such that

$$
\begin{aligned}
& U_{L}(r)=-\int_{0}^{r} F(r) \mathrm{d} r .=-\left\{\int_{0}^{\Delta} F(r) \mathrm{d} r+\int_{\Delta}^{2 \Delta} F(r) \mathrm{d} r+\ldots . . \int_{r-\Delta}^{r} F(r) \mathrm{d} r\right\}, \text { where } \\
& \int_{i \Delta}^{(i+1) \Delta} F(r) \mathrm{d} r=\mu_{G} \int_{i \Delta}^{(i+1) \Delta} \frac{I_{i} I_{i+1}}{2 \pi \Delta} 2 \pi r_{i} \mathrm{~d} r,
\end{aligned}
$$

with $I_{i}=v_{t} \rho_{i} \Delta\left[\mathrm{kg} \mathrm{s}^{-1}\right]$,

where $v_{t}$ is the tangential velocity of the massive objects and where $\rho(r)$ is the mass density (in $\mathrm{kg} / \mathrm{m}^{2}$ ) in the spiral arms. The observer's position here and elsewhere in this analysis, is assumed being in the center of the galaxy. In the case that the orbit of the massive object under consideration is circular, the tangential velocity $v_{t}$ is the same as the transverse velocity $v$ as defined in (1). In non-circular orbits, the value of the latter one decreases with $r$ as a consequence of the conserved angular momentum as defined by (4). It has to be noted, though, that this conservation law only holds within an orbit under consideration. It does not apply when comparing massive objects in different orbits. Experimental observation reveals that $v_{t}$ is more or less independent of $r$. So, let us continue under this assumption and let us investigate if a consistent theory can be built that is not in conflict with it.

Further analysis reveals

$$
\int_{i \Delta}^{(i+1) \Delta} F(r) d r=\mu_{G} \int_{i \Delta}^{(i+1) \Delta} \frac{I_{i} I_{i+1}}{2 \pi \Delta} 2 \pi r_{i} \mathrm{~d} r=\mu_{G} \int_{i \Delta}^{(i+1) \Delta} \frac{\left(v_{t} \rho_{i} \Delta\right)^{2}}{\Delta} r_{i} \mathrm{~d} r=\mu_{G} v_{t}^{2} r_{i} \rho_{i}^{2} \Delta^{2}
$$

Under use of the definitions $\varepsilon_{G}=1 / 4 \pi G$ and $c_{G}=\left(\sqrt{\varepsilon_{G} \mu_{G}}\right)^{-1}$, it follows from (5) and (7),

$$
U_{L}(r)=-\mu_{G} v_{t}^{2} \Delta^{2} \sum_{i=1}^{N} r_{i} \rho_{i}^{2}=-\frac{4 \pi G}{c_{G}^{2}} v_{t}^{2} \Delta^{2} \sum_{i=1}^{N} r_{i} \rho_{i}^{2}
$$

as the total potential energy of the $N^{\text {th }}$ spiral arm due to the gravitational Lorentz force. 
Per unit of mass, the contribution is

$U_{L}^{\prime}(r)=\frac{1}{2 \pi r \rho_{N} \Delta} U_{L}(r)=-\frac{2 G v_{t}^{2} \Delta}{c_{G}^{2} r} \sum_{i=1}^{N} \frac{r_{i} \rho_{i}^{2}}{\rho_{N}}=\frac{G v_{t}^{2}}{\pi c_{G}^{2} r} M_{r s}$,

with $\quad M_{r s}=2 \pi \Delta \sum_{i=1}^{N} \frac{r_{i} \rho_{i}^{2}}{\rho_{N}}$.

It will be clear that $M_{r s}$ has about the same value as the sum $M_{r}$ of mass in the $N$ spiral arms within the radius $r$ under consideration.

From (3) and (8), we have, without Lorentz force

$\frac{1}{2} m v_{t}^{2}=\frac{m G M_{r}}{r}$

and with Lorentz Force,

$\frac{1}{2} m(1-\alpha) v_{t}^{2}=\frac{m G M_{r}}{r}$, where

$\alpha=\frac{2}{\pi c_{G}^{2}} \frac{G M_{r s}}{r}$.

It will be clear that if $\alpha$ would have a substantial value, it would have a substantial influence on the tangential velocity $v_{t}$ of the star in the spiral arms rotating around the center of the galaxy. Note that $G M_{r} / r$ has the dimension of a squared velocity. As long as $\alpha<1$, equation (12) is consistent with a circular orbit equation. The circular motion would change into a spiral one if $\alpha$ exceeds 1 . If $\alpha$ is about 0.9 , it would seem as if the central mass $M_{r}$ is raised to a tenfold. It would seem as if $90 \%$ of its mass is "dark". Depending on the radial dependency of $M_{r}$ and on the value $(1-\alpha)$, it might well be that $v_{t}$ will not only show a flat behavior as a function of $r$, but that it will be substantially larger than expected from the amount of visible mass as well.

So, the crucial question is whether $\alpha$ may have a substantial value. It will be clear that if $c_{G}$ would equate the light velocity in empty space, gravimagnetism cannot be the substitute for dark matter. So, the crucial question is if there is a reason to suppose that $c_{G}$ can be different from the light velocity. This issue will be further addressed in the next paragraph. Before doing so, I wish to point to a seeming anomaly. In this analysis it has been taken for granted that the velocity curve of moving objects in the galaxy is flat, implying that the tangential velocity of orbiting objects is independent of the distance to the center. This corresponds with experimental evidence. It means, though, that the objects are revolving in a circle. The structure of the galaxy, however, shows spirals. The implication is that stellars cannot maintain their position in the spiral arms. If they did, their velocity should increase with the radius, because it would seem as if they were part of a rigid body. This seems being a paradox. In fact, it is not, because the spirals have to be considered as a pattern of mass 
density, which for some reason, is created in the dark initial phase of the galaxy. So, where the spiral arms with their high mass density preserve their position in the galaxy, individual stars don't. The spiral arms have to be considered as traffic jams, where stellars move in and move out. If this were not the case, the flatness of the velocity curve would demolish the spiral characteristic.

\section{General Relativity and gravimagnetism}

The resemblance between the electromagnetic field and the gravitational field does not necessarily mean that the one is a Chinese copy of the other. One thing is, for instance, the fact that where in electromagnetism charges are bipolar, charges in gravity are unipolar. Where the vectorial character of the electromagnetic field's potential function is without any doubt, it might still be that the potential function of the gravitational field is scalar. The observation that the weak field limit of the gravitational field, as expressed by Einstein's field equation, gives rise to a wave equation, does not necessarily imply that the gravity field's potential function is vectorial. To provide more insight in this, it might be useful to compare the origin of Maxwell's wave equation with the gravity wave equation that results from Einstein's Field Equation. Maxwell's wave equation is straightforwardly derived from the four well known Maxwell equations, written in SI notation as,

$\nabla \cdot \mathbf{E}=\rho / \varepsilon_{0} ; \nabla \cdot \mathbf{B}=0 ; \nabla \times \mathbf{E}=-\partial \mathbf{B} / \partial t$ and $\nabla \times \mathbf{B}=\mu_{0}\left(\mathbf{j}+\varepsilon_{0} \partial \mathbf{E} / \partial t\right)$

Combining the time derivatives of these equations followed by elementary algebraic manipulation, results in the well known Maxwellian wave equations for the electric field strength $\mathbf{E}$ and the magnetic field strength $\mathbf{B}$, which are spatially and temporally of second order. These fields are created as a consequence of a spatial charge $\rho$ (which may reduce to a Dirac type distribution) and a current density $\mathbf{j}$.The Maxwell equations as such are not sufficient for describing mechanical forces on charged particles. As soon as a force $F$ is assigned on a particle with electrical charge $q$ as $F=q E$, Einstein's description of Maxwell's laws in terms of special relativity prescribes the generalization of the force by including the Lorentz force as a consequence of a moving charge $q$ with velocity $v$ as

$\mathbf{F}=q \mathbf{E}+q \mathbf{v} \times \mathbf{B}$

The Faraday induction $\partial \mathbf{B} / \partial t$ and the displacement current $\varepsilon_{0} \partial \mathbf{E} / \partial t$, which mutually couple a magnetic field with an electric field, are the essential ingredients to generate the wave equation. This evokes the question in how far the bipolarity of electric charges in the source terms $\rho / \varepsilon_{0}$ and $\mathbf{j}$ is responsible for these essential ingredients. Anyhow, where the displacement current in electromagnetism can be readily understood from the interaction between positive and negative charges in the interrupt of a charged conductor by a condenser, there is not such equivalence available with unipolar charges in gravity. Whether a one-to-one mapping of the electromagnetic Maxwellian equations to gravity is allowed is an open question still. This holds in particular for the dynamic terms $\varepsilon_{0} \partial \mathbf{E} / \partial t$ (displacement current) and $\partial \mathbf{B} / \partial t$ (Faraday induction). It might well be that these ingredients are absent in gravimagnetism. 
Let us now consider the gravitational wave equation as a consequence of the weak field limit of the Einsteinean Field Equation. The equation reads as,

$G_{\mu v}=\frac{8 \pi G}{c^{4}} T_{\mu \nu} \quad$ with $G_{\mu v}=R_{\mu \nu}-\frac{1}{2} R g_{\mu v}$

where $T_{\mu \nu}$ is the stress-energy tensor, which describes the energy and the momenta of the source(s) and where $R_{\mu \nu}$ and $R$ are respectively the so-called Ricci tensor and the Ricci scalar, which can be calculated if the metric tensor components $g_{\mu \nu}$ are known [6,7]. In the case that a particle under consideration is subject to a central force only, the time-space condition shows a rotational symmetric isotropy. This allows to read the metric elements $g_{i j}$ from a simple line element that can be written as

$\mathrm{d} s^{2}=g_{00}(r, t) \mathrm{d} q_{0}^{2}+g_{r r}(r, t) \mathrm{d} r^{2}+r^{2} \sin ^{2} \vartheta \mathrm{d} \varphi^{2}+r^{2} \mathrm{~d} \vartheta^{2}$,

where $q_{0}=\mathrm{i} c t$ and $\mathrm{i}=\sqrt{-1}$.

It means that the number of metric elements $g_{i j}$ reduce to a few, and only two of them are time and radial dependent. In the weak field limit, we have

$g_{00}(r, t)=1+h_{00}(r, t)$, where $\left|h_{00}(r, t)\right|<<1$, and

$g_{r r}(r, t)=1+h_{r r}(r, t)$, where $\left|h_{r r}(r, t)\right|<<1$.

Under this condition, Einstein's Field Equation can be evaluated into a wave equation (see Appendix A),

$\frac{\partial^{2} h_{00}}{\partial r^{2}}-\frac{1}{c^{2}} \frac{\partial^{2} h_{00}}{\partial t^{2}}=0$ and $\frac{\partial^{2} h_{r r}}{\partial r^{2}}-\frac{1}{c^{2}} \frac{\partial^{2} h_{r r}}{\partial t^{2}}=0$, with $\frac{\partial h_{00}}{\partial r}=-\frac{\partial h_{r r}}{\partial r}$

Obviously, a central force from a sudden source in the center of empty space is enough to create a gravitational wave function. This wave function propagates with the vacuum light velocity, thereby proving the causality of the gravitational force. It was Karl Schwarzchild, who calculated the numerical value for the metric elements for the case that the central force is Newton's gravity force from a central mass $M$. In that case

$h_{r r}=\frac{2(G M / r)}{c^{2}}$ and $h_{00}=-\frac{2(G M / r)}{c^{2}}$.

So far in these considerations, possible rotation energy of the source in the center, has not been considered. Inclusion of it will, of course, affect the metric tensor. It makes it more complex, because one might expect that the nice symmetric isotropy will be lost. Generically, the line element has to be expressed in terms of the Kerr metric [8], which in the canonical gravimagnetic formalism is simplified to [9] 


$$
\mathrm{d} s^{2}=-c^{2}\left(1-2 \frac{\Phi}{c^{2}}\right) \mathrm{d} t^{2}-\frac{4}{c}(\mathbf{A} \cdot . \mathrm{d} \mathbf{x}) \mathrm{d} t+\left(1+2 \frac{\Phi}{c^{2}}\right) \delta_{i j} \mathrm{~d} x^{i} \mathrm{~d} x^{j}
$$

where $\delta_{i j}$ is the Kronecker delta and where, far from the source,

$\Phi=\frac{G M}{r}, \mathbf{A}=\frac{G}{c} \frac{\mathbf{J} \times \mathbf{x}}{r^{3}}, r=|\mathbf{x}|$ and where $\mathbf{J}$ is the angular momentum of the source. As will be shown later in this text, this metric is the basis for a canonic formulation of the gravimagnetic equation set, which will appear somewhat different from a naïve transposition of the Maxwellian set that we shall pursue first.

In the special case that a particle subject to this metric is revolving in the equatorial plane of the source, the influence of the source's angular momentum becomes manifest as a quasi Lorentz force, which can be modeled as an add-on to the central force. Under this condition the nice symmetric isotropy is restored. It will be clear that the value of the proportionality constant $\mu_{G}$ has a large impact on the strength of the quasi Lorentz force. It is quite common to establish this value from the following observation. First of all, Poisson's law for gravity is invoked, which states

$\nabla^{2} \Phi=4 \pi G \rho$

In analogy with Poisson's law for electromagnetism a gravity equivalent $\varepsilon_{G}$ for electric permeability is defined, such that

$\varepsilon_{G}=1 / 4 \pi G$

The next step is relating

$c^{2}=\frac{1}{\varepsilon_{0} \mu_{0}} \Leftrightarrow c_{G}^{2}=\frac{1}{\varepsilon_{G} \mu_{G}} ?$

The justification for equating $c^{2}$ with $c_{G}^{2}$, as is common practice, is the statement that the presence of a vector potential associated with the angular momentum brings the causality of the gravity field such that it propagates with the vacuum light velocity. The presence of the angular momentum is not required for bringing the causality. As proven in the Appendix, the causality is implicitly provided by Einstein's Field equation even in the absence of an angular momentum of the source. Therefore, causality of the gravity field is no reason to equate $c^{2}$ with $c_{G}^{2}$.

Gravimagnetism might be essentially different from electromagnetism in the sense that the mutual coupling between a gravitational equivalent of an electric field generated by a scalar potential and a gravitational equivalent of a magnetic field generated by a vector potential might be absent. The two types of fields might exist, however, maybe without mutual coupling. Without the coupling, the quantity $c_{G}$ is just a constant that has nothing to do with radiation or propagation of gravitational energy by a Poynting vector. Arguments for believe 
in the absence of this coupling is given above, where the role of the difference in polarity of charges has been discussed. Let us continue by estimating the value of $\mu_{G}$ from known experiments. This will be done in the next paragraph.

\section{Implications from the Gravity B Probe experiment}

Probably the most relevant experiment for establishing the value of the gravimagnetic constant $\mu_{G}$ has been offered by the Gravity B Probe project $[10,11]$. This project has been set up to compare theoretical values of two gravitational effects with experimental evidence. These effects are, respectively, the geodetic effect and frame dragging (also known as the Lense-Thirring effect $[12,13])$. The geodetic effect is related with the phenomenon of the perihelium shift of planets in the solar system. This shift is predicted by Einstein's General Relativity. The perihelium of a planet in an almost circular loop with radius $r_{0}$ shifts each revolution by an amount of $[7,14]$

$\Delta \varphi^{\prime}=\frac{3 \pi R_{S}}{r_{0}}$, where $R_{S}=\frac{2 M_{s o l} G}{c^{2}}$

The quantity $R_{S}$ is known as the Schwartzschild radius. A satellite orbiting around the earth will show the same effect. It can be measured by a gyroscope on board of a satellite with its axis pointing to a cosmological object in deep space. As a consequence of the curving of space-time due to the energetic gravitational field of the earth, the gyroscope on board of the satellite will show a precession motion. One might think that, per revolution of the satellite at a radius $r_{\text {, }}$ around the earth enter, the angle of the gyroscope axis will shift by an amount that equals the value of the perihelium shift. Actually, it is more complicated than that. The theoretical value of the perihelium shift is calculated from the line element of an isotropic non-rotating metric of the type as given by (17). More particularly,

$\mathrm{d} s^{2}=-\left(1-\frac{R_{s}}{r}\right) \mathrm{d} t^{2}+\left(1-\frac{R_{s}}{r}\right)^{-1} \mathrm{~d} r^{2}+r^{2} \sin ^{2} \vartheta \mathrm{d} \varphi^{2}+r^{2} \mathrm{~d} \vartheta^{2}$

The calculated value of the perihelium shift nicely fits with experimental evidence from orbiting planets indeed. However, as noted before, this line element is incomplete, because the influence of the rotation energy of the central mass (of the sun for planets or the earth for orbiting satellites) is not included. Nevertheless, the perihelium shift behaves as predicted by (25). This can be understood as follows. The influence of the rotation energy can be accounted for by two different mechanisms. These mechanisms are physically equivalent, but are different in modeling. The first one is changing the line element of field metric followed by an analysis of the angular momentum of a mass object moving in this field. The second one is leaving the field metric unchanged and modeling the influence of the rotation energy as the add-on of a gravimagnetic field. In the latter model, a gravitational Lorentz force is associated with this field and as long as the revolving of a massive object takes place in the equatorial plane of a rotating central mass, the gravitational Lorentz force is central. This force is just a slight add-on on the Newtonian force, thereby hardly affecting the amount of perihelium shift of a revolving massive object in the equatorial plane. The angle of the axis of a gyroscope placed in a satellite orbiting in the equatorial plane of the earth will show per orbit a slight additional amount of phase shift in the equatorial plane. 
Later in this article, l'll show that this additional amount is virtually indistinguishable from the amount as predicted by the perihelium shift of a non-rotating metric. For that reason the perihelium shift of planets is not different from the one as calculated from the Schwarzschild metric (26). In fact, the only thing to be done is to relate the radius of Mercury's orbit and the solar mass with the radius of the Gravity B satellite $(\approx 7000 \mathrm{~km})$ and the earth mass. This gives 13188 macs/yr (milliarcsec per year). Curiously, this is about twice the value as reported from the Gravity B project $[10,11]$. The reason of the difference will be discussed later in this text.

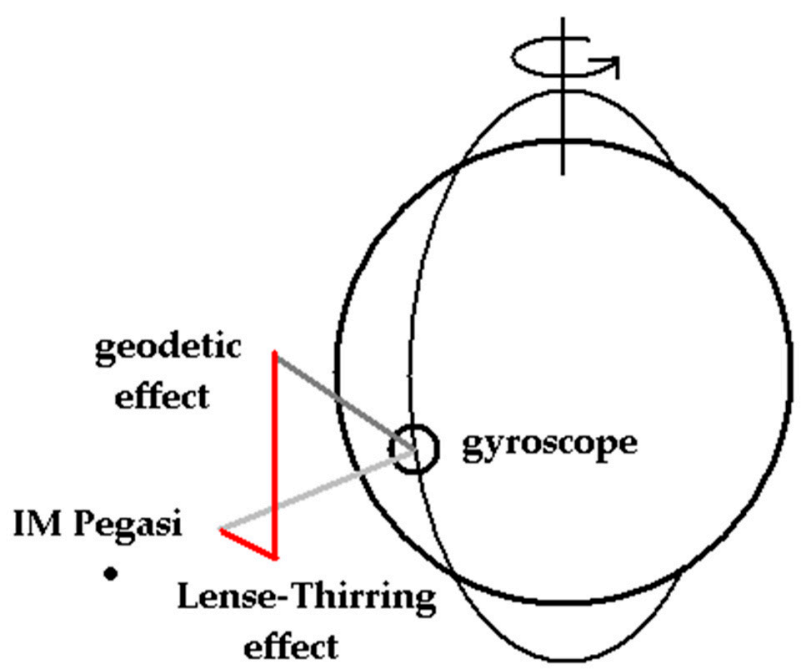

Figure 4. The two orbital precession influences (perihelium shift/geodetic effect and Lorentz force/LenseThirring effect) are coherent (i.e. in the same direction) for massive objects in an equatorial plane and noncoherent for massive objects in a polar plane. IM Pegasi is the guide star for the gyroscope. From: large.stanford.edu/courses/2007/ph210

This picture will change in the case that the satellite with gyroscope(s) orbits in the polar plane. A gyroscope is a device that always keeps its orientation, irrespective of the bottom on which it is placed. If, for some reason whatever, the bottom tilts or turns, the orientation the gyroscope's axis is preserved. For an observer fixed to the bottom, however, it seems as if the gyroscope's axis shifts (precession). A particular example is Foucault's pendulum. The earth rotates under the plane of swinging. For the observer on earth it seems as if the pendulum plane rotates. The Lorentz force on a massive object orbiting in a polar plane is no longer oriented in the same direction as the gravitational force. The force is maximum and orthogonal to the gravitational force when the satellite passes the poles and the force is zero when the satellite passes the equator. As a consequence the orientation of the plane of orbit will be subject to a spatial phase shift orthogonal to the polar plane. This will be experienced by an observer in the satellite as a precession of the gyroscope next to the orbital perihelium phase shift. Figure 4 illustrates the process. Let us first consider the gravimagnetic view.

\section{The gravimagnetic view}

In line with the reflections on gravimagnetism earlier in this article, I wish to develop the gravimagnetic view by conceiving mass particles as charged particles with charge $m$. I wish to do so on the basis of the naive analogy as summarized in Table II. The correspondence/difference with the canonical view will be discussed later. A large massive volume will be modeled as a massive body with some internal mass density $\rho$. As a 
consequence of this mass density, a gravimagnetic field is generated, similar to the magnetic field of a volume with some electrical space charge. According to this picture, the earth will be modeled as a sphere with a uniform mass density $\rho$. The calculation of the generated (gravi)magnetic field $\mathbf{B}_{\mathbf{G}}$ is not trivial. The field for a sphere with uniform surface charge density $\sigma$ can be found in textbooks [15]. It appears that,

$\mathbf{B}_{\mathbf{G}}=\frac{\mu_{G} \omega \sigma}{3} \frac{R^{4}}{r^{3}}\{2 \cos \vartheta \hat{\mathrm{r}}+\sin \vartheta \hat{\vartheta}\}$, for $r \geq R$

$(\cos \vartheta \mathbf{r}=\cos \vartheta \sin \vartheta \mathbf{x}+\cos \vartheta \cos \vartheta \mathbf{z} ; \sin \vartheta \hat{\vartheta}=-\sin \vartheta \cos \vartheta \mathbf{x}-\sin \vartheta \sin \vartheta \mathbf{z})$

where $\omega$ is the angular velocity of the earth rotation and where $\hat{\mathbf{r}}$ and $\hat{\vartheta}$, respectively, are unit vectors in $r$-direction and in $\vartheta$-direction. This can be converted into the field of a sphere with uniform volume charge density $\rho$ by integrating over shells $\rho \mathrm{d} r=\sigma$, so that

$\mathbf{B}_{\mathrm{G}}=\frac{\mu_{G} \omega \rho}{3 r^{3}} \frac{R^{5}}{5}\{2 \cos \vartheta \hat{\mathrm{r}}+\sin \vartheta \hat{\vartheta}\}$, for $r \geq R$

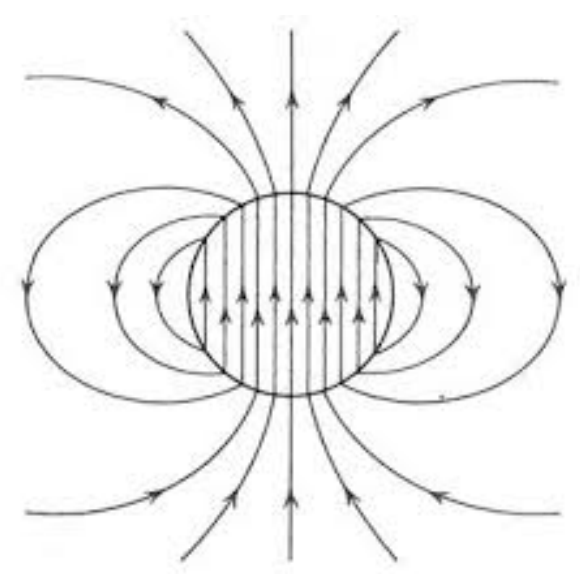

Figure 5. The rotational symmetry of the gravimagnetic field strength B.

In terms of the peripheral equatorial speed $v_{e}=\omega R_{e}$ and the mass $M_{e}=4 \pi \rho R_{e}^{3} / 3$, the magnitude of the equatorial magnetic field $(\vartheta=\pi / 2)$ can be expressed as

$B_{G}=\frac{\mu_{G}}{20 \pi} \frac{R^{3}}{r^{3}} \frac{M_{e}}{R_{e}^{2}} v_{e}$

As a consequence of this gravimagnetic field, a massive object with mass $m$, orbiting in the equatorial plane with tangential velocity $v$, is subject to a Lorentz force, the magnitude $F_{L}$ of which is determined from $m \mathbf{v} \times \mathbf{B}_{\mathbf{G}}$ as

$$
F_{L}=m \frac{\mu_{G}}{20 \pi} \frac{R_{e}^{3}}{r^{3}} \frac{M_{e}}{R_{e}^{2}} v v_{e}=m \frac{M_{e} G}{5 R_{e}^{2}} \frac{R_{e}^{3}}{r^{3}} \frac{v_{e} v}{c_{G}^{2}}
$$


In the equatorial plane, this force is balanced by a centripetal force, so that

$$
\begin{aligned}
\frac{m v^{2}}{r} & =m \frac{M_{e} G}{5 R_{e}^{2}} \frac{R_{e}^{3}}{r^{3}} \frac{v_{e} v}{c_{G}^{2}} \rightarrow v=r \frac{M_{e} G}{5 R_{e}^{2}} \frac{R_{e}^{3}}{r^{3}} \frac{v_{e}}{c_{G}^{2}} \rightarrow \\
\frac{\mathrm{d} \varphi}{\mathrm{d} t} & =\frac{M_{e} G}{5 R_{e}^{2}} \frac{R_{e}^{3}}{r^{3}} \frac{v_{e}}{c_{G}^{2}}
\end{aligned}
$$

This gives per orbit an additional shift on top of the perihelium shift to the amount of

$$
\Delta \varphi=\frac{2 \pi}{5} \frac{M_{e} G}{c_{G}^{2} R_{e}} \frac{R_{e}^{2}}{r^{2}} \frac{v_{e}}{v}=\frac{2 \pi}{5} \frac{M_{e} G}{c_{G}^{2}} \frac{R_{e}}{r^{2}} \frac{v_{e}}{v}=\frac{\pi}{5} \frac{R_{S G}}{r}\left(\frac{R_{e}}{r} \frac{v_{e}}{v}\right)
$$

with $R_{S G}=\frac{2 M_{e} G}{c_{G}^{2}}$.

If $c_{G}$ is equated with the vacuum light velocity $c, R_{S G}$ is the same as the Schwarzschild radius.

It will be clear that if this is the case, this additional shift as a consequence of the rotation of the central mass is negligible for planets in orbits around the sun, because of the large mismatch between the radius of the sun and the average radius of the orbit. This is different for a satellite orbiting in the periphery of the earth.

In the Gravity Probe B project the distance of the satellite to the earth center amounts to $r=7000 \mathrm{~km}$. The peripheral speed of the earth is $v_{e}=2 \pi \times 6400 /(24 \times 60 \times 60) \mathrm{km} / \mathrm{s}$. From these expressions is straightforwardly calculated that, under assumption of $c_{G}=c$, this additional precession over a year amounts to 50 macs, while, as noted before, the perihelium shift amounts to $13188 \mathrm{macs} / \mathrm{yr}$ (milliarcsec per year). Note that the perihelium shift is not dependent on $c_{G}$, but on $c$ instead.

Let us now suppose that the satellite orbits in the polar plane. Now the satellite experiences a Lorentz force orthogonal to the orbit plane. From (28) it is obvious that the Lorentz force while passing the poles is twice as large as in the equatorial case. It is zero while passing the equator. In other positions, the strength of the Lorentz force is composed as

$F_{L}=B_{z} v_{x}+B_{x} v_{z}$, where

the two components of the gravimagnetic field strength are given by,

$B_{z}=B_{G}(2 \cos \vartheta \cos \vartheta-\sin \vartheta \sin \vartheta)$ and $B_{x}=B_{G}(2 \cos \vartheta \sin \vartheta-\sin \vartheta \cos \vartheta)$,

and where the tangential velocity $v$ is decomposed as 
$v_{z}=-v \sin \vartheta$ and $v_{x}=v \cos \vartheta$

Figure 6 shows these quantities as a function of $\vartheta$.
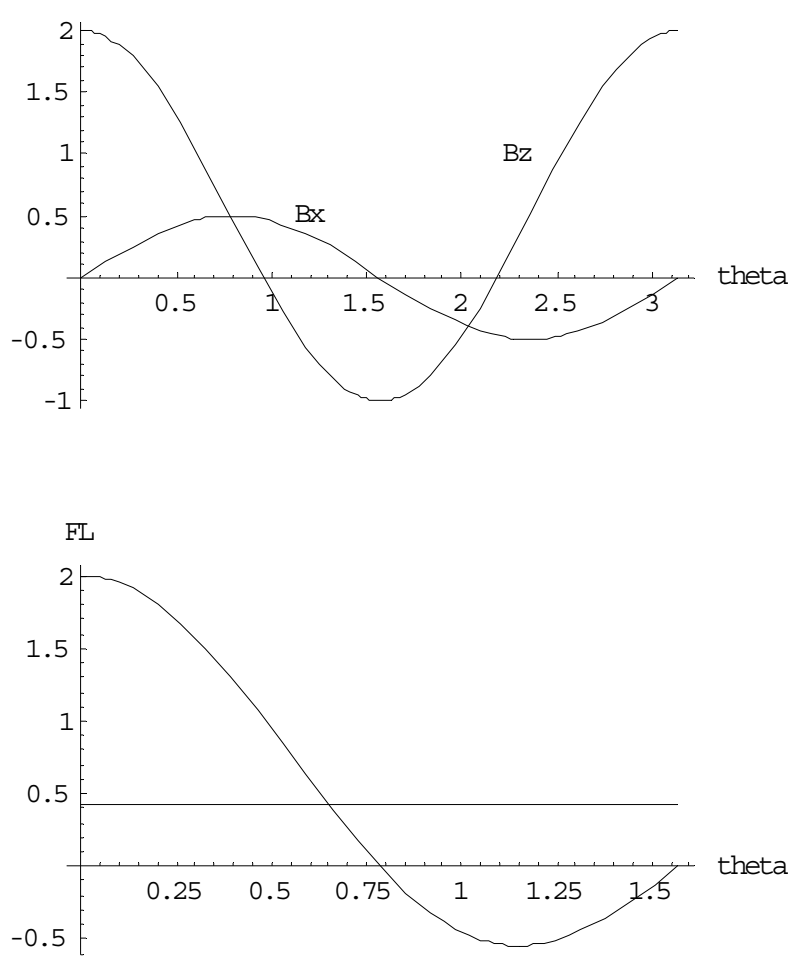

Figure 6. Upper part: The $\mathrm{z}$ and $\mathrm{x}$ component of the gravimagnetic fieldstrength $\mathrm{B}$ as a function of $\vartheta$ (the $\mathrm{y}$ component is zero). Lower part: The Lorentz force as a function of $\vartheta$ and its average value (0.42).

The Lorentz force is orthogonal to the plane of motion. Averaged over the orbit, the effective Lorentz force is $0.42 \mathrm{x}$ in magnitude as in the equatorial case (thereby resulting in a frame dragging effect of 21 macs/yr). Now, however, the phase shift is orthogonal to the perihelium phase shift. The labor executed by the Lorentz force is converted into the energy of a precession motion orthogonal on the plane of motion. In the equatorial case the two precessions are coherent, i.e. pointing in the same direction, while they are incoherent in the polar case. In the latter case observation of the gyroscope allows to measure the two effects independently.

The reported measurement data from the Gravity B probe project are, respectively, for the geodetic effect, 6606.1 macs/yr (milliarcsec per year), and for the frame dragging effect 37.2 macs/yr. Curiously, where, as already noted, the figure for the geodetic effect is almost exactly a factor 2 smaller than results from the analysis above of the perihelium shift, it is about a factor 2 larger for the gravimagnetic effect. The reasons for these discrepancies will be discussed below.

\section{Schiff's view}

The gravity B probe project has been set-up for testing the gravity theory as documented by L.I. Schiff in his classic article [16] . This theory describes the influence of a rotatic metric, 
such as produced by the earth, on the spin vector (= angular momentum vector) $\mathbf{S}$ of a gyroscope placed in an orbiting satellite. The conclusion of the theory is that the time behavior of the spin vector from the perspective of an observer in the satellite and corrected for the earth time $t$, can be expressed as

$$
\frac{\mathrm{d} \mathbf{S}}{\mathrm{d} t}=\mathbf{\Omega} \times \mathbf{S}
$$

where

$\boldsymbol{\Omega}=\mathbf{\Omega}_{\mathbf{G}}+\mathbf{\Omega}_{\mathbf{F D}}=\frac{3 G M}{2 c^{2} r^{3}}(\mathbf{r} \times \mathbf{v})+\frac{G I}{c^{2} r^{3}}\left\{\frac{3}{r^{2}}(\boldsymbol{\omega} \cdot \mathbf{r}) \mathbf{r}-\boldsymbol{\omega}\right\}$.

where $I$ is the earth's moment of inertia. The first term is independent of the earth's rotation, while the second term determines its impact. In terms of the earth's angular momentum $\mathbf{J}=I \boldsymbol{\omega}$, this can be rewritten as,

$\boldsymbol{\Omega}=\boldsymbol{\Omega}_{\mathbf{G}}+\boldsymbol{\Omega}_{\mathbf{F D}}=\frac{3 G M}{2 c^{2} r^{3}}(\mathbf{r} \times \mathbf{v})+\frac{G}{c^{2} r^{3}}\left\{\frac{3}{r^{2}}(\mathbf{J} \cdot \mathbf{r}) \mathbf{r}-\mathbf{J}\right\}$

The energy $E_{G}$ associated with the first term amounts to

$$
E_{G}=\mathbf{S} \cdot \mathbf{\Omega}_{\mathbf{G}}=m r^{2} \frac{\mathrm{d} \varphi}{\mathrm{d} t} \frac{3 G M}{2 c^{2} r^{2}} v
$$

This energy is converted into a rotational precession energy $E_{p}$, so that

$$
E_{p}=E_{G} \rightarrow \frac{1}{2} J \omega^{2}=\frac{1}{2} r^{2} m\left(\frac{\mathrm{d} \varphi}{\mathrm{d} t}\right)^{2}=m r^{2} \frac{\mathrm{d} \varphi}{\mathrm{d} t} \frac{3 G M}{2 c^{2} r^{2}} v=m r^{2} \frac{\mathrm{d} \varphi}{\mathrm{d} t} \frac{3}{4} R_{s} \frac{1}{r^{2}} v
$$

and so

$$
\frac{\mathrm{d} \varphi}{\mathrm{d} t}=\frac{3 R_{s}}{2 r^{2}} v=\frac{3 R_{s}}{2 r^{2}}\left(\frac{2 \pi r}{\Delta T}\right)
$$

Per orbit of the satellite, it results in a phase shift $\Delta \varphi_{G}$ to the amount of

$$
\Delta \varphi_{G}=\frac{3 \pi R_{s}}{r}
$$

This is the same as the value of the perihelium shift defined in the previous paragraph. For the second term precession energy of a gyroscope in the equatorial plane, we have from (36)

$$
E_{G}=\mathbf{S} \cdot \mathbf{\Omega}_{\mathbf{F D}}=m r \frac{\mathrm{d} \varphi}{\mathrm{d} t} \frac{G}{c^{2} r^{3}} J
$$


For the angular momentum of the earth, interpreted as a spherical object, we have

$J=M_{e} \frac{4 \pi R_{e}^{2}}{5 T_{e}}=M_{e} \frac{4 \pi R_{e}^{2}}{5 T_{e}}=\frac{2}{5} M_{e} R_{e} v_{e}$

where $T_{e}$ is the rotation period of the earth.

With these expressions the second term of the gyroscope precession in the equatorial plane results from

$$
E_{p}=E_{G} \rightarrow \frac{1}{2} r^{2} m\left(\frac{\mathrm{d} \varphi}{\mathrm{d} t}\right)^{2}=m r^{2} \frac{\mathrm{d} \varphi}{\mathrm{d} t} \frac{G}{c^{2} r^{3}} \frac{2}{5} M_{e} R_{e} v_{e}=\frac{1}{5} m r^{2} \frac{\mathrm{d} \varphi}{\mathrm{d} t} R_{s} \frac{R_{e} v_{e}}{r^{3}}
$$

so that

$$
\frac{\mathrm{d} \varphi}{\mathrm{d} t}=\frac{2}{5} R_{s} \frac{R_{e} v_{e}}{r^{3}}
$$

and therefore

$$
\Delta \varphi_{G}=\frac{2}{5} R_{s} \frac{R_{e} v_{e}}{r^{3}}\left(\frac{2 \pi r}{v}\right)=\frac{4 \pi R_{s}}{5 r} \frac{R_{e} v_{e}}{r v} \text { per orbit of the satellite. }
$$

This gives $200 \mathrm{macs} / \mathrm{yr}$. It is $4 \mathrm{x}$ larger than in the gravimagnetic view for $c_{G}=c$. A gyroscope in a polar plane shifts $(200 \times 0.42=) 84$ macs/yr. Similar as the perihelium shift, it is $2 \times$ larger than quoted from the Gravity B probe project. The reason for this discrepancy has to do with the semantics of $\boldsymbol{\Omega}_{\mathrm{FD}}$. Although the dimensionality of $\boldsymbol{\Omega}_{\mathbf{F D}}$ is $\left[\mathrm{s}^{-1}\right]$, it should not be interpreted as $\mathrm{d} \varphi / \mathrm{d} t$. In fact $\boldsymbol{\Omega}_{\mathrm{FD}}$ represents the gravimagnetic field, which has a dimensionality of $\left[\mathrm{s}^{-1}\right]$ as well (see Table II). This implies that the product $\mathbf{S} \cdot \mathbf{\Omega}$ is an energy. Equating this energy with the rotational energy of the gyroscope, we get,

$$
\frac{1}{2} r^{2} m\left(\frac{\mathrm{d} \varphi}{\mathrm{d} t}\right)^{2}=r^{2} m \frac{\mathrm{d} \varphi}{\mathrm{d} t} \Omega_{F D} \rightarrow \frac{\mathrm{d} \varphi}{\mathrm{d} t}=2 \Omega_{F D}
$$

This makes the $\mathrm{d} \varphi / \mathrm{d} t$ twice the value of $\boldsymbol{\Omega}_{\mathrm{FD}}$. It might be instructive here to note that other authors, e.g. lorio [17], have expressed the Lense-Thirring formulae as $\dot{\Omega}_{L T}$, i.e, with a "dot". These formulae represent $\mathrm{d} \varphi / \mathrm{d} t$ and have twice the value as Schiff's $\boldsymbol{\Omega}_{\mathrm{FD}}$ indeed. It is therefore quite probable that the measurement data reported from the Gravity B project apply to $\boldsymbol{\Omega}$ instead to $\mathrm{d} \varphi / \mathrm{d} t$.

\section{Comparing Schiff's theory with gravimagnetism}

The result of the Gravity B probe experiment confirms Schiff's conclusion about the weak limit of Einstein's Field Equation. Compared with the gravimagnetic view, there is no difference in the geodetic part, which, in a proper interpretation of the reported data, is the same as the well known perihelium shift of planetary objects in the solar system. The frame 
dragging result in the weak limit is exactly $4 x$ larger than follows from a one-to-one transposition of Maxwellian laws to gravity. Repairing this discrepancy requires a modification of the Lorentz force by a factor 4 . This can be done in the naive view on gravimagnetism by accepting $\mu_{G}=16 \pi G / c^{2}$, implying that $c^{2} \neq c_{G}^{2}$. The alternative approach is to derive a canonical formulation of the gravimagnetism that does not show the discrepancy, while sticking to $c^{2}=c_{G}^{2}$ [9]. Starting point is the metric shown in (21). This allows a derivation for the field's Lagrangian density, which executes a (Lorentz) force on a test particle with mass $m$ to the amount of

$\mathbf{F}=m \mathbf{E}+(2 m) \mathbf{v} \times \mathbf{B}$,

where, under stationary conditions, i.e. $\partial \mathbf{A} / \partial t=0$, the gravimagnetic quantities $\mathbf{E}$ and $\mathbf{B}$ are related with the quantities in the metric tensor (21) as

$\mathbf{E}=-\nabla \Phi$ and $\mathbf{B}=\nabla \times \mathbf{A}$

Comparing (15) with (46), it is obvious that the factor 4 is not yet fully accommodated. To do so, Mashhoon has proposed to modify the naïve Maxwellian transposition to

$\nabla \cdot \mathbf{E}=\rho / \mathcal{E}_{G} ; \nabla \cdot(\mathbf{B} / 2)=0 ; \nabla \times \mathbf{E}=-\partial(\mathbf{B} / 2) / \partial t$ and $\nabla \times(\mathbf{B} / 2)=\mu_{G}\left(\mathbf{j}+\varepsilon_{G} \partial \mathbf{E} / \partial t\right)$.

It will be obvious from the last member of this set that this doubles the gravimagnetic component $\mathbf{B}$. This doubling together with the term $2 m$ in (46) makes the gravimagnetic equation set (46-48) compatible with experimental evidence. It has to be noted, however, that where the increase of the Lorentz force by a factor 4 straightforwardly follows from the metric, the gravitational equivalents for the Faraday induction and the displacement current are heuristically introduced to obtain symmetry between gravimagnetism and electromagnetism. It might well be that the gravitational field is not radiating and that a spin-1 graviton, such as suggested by the equation set, is non-existing.

\section{Cosmological Gravity}

The experimental results of the Gravity B project have shown that gravimagnetism exists, but that it is inadequate to explain the origin of black matter, because of the weakness of the frame dragging effect. So, something else is required to explain the phenomenon of flat rotation curves in a galaxy. Unusual problems ask for unusual explanations. In this paragraph, I wish to propose an unusual explanation, albeit that use will be made of common concepts in field theory. To this end, the Lagrangian density of the cosmological gravity field will be derived from the generic expression,

$\odot=-\frac{1}{2} \partial_{\mu} \Phi \partial^{\mu} \Phi+U(\Phi)+\rho \Phi$

where $U(\Phi)$ is the potential energy of the field and where $\rho \Phi$ is the source term. Let us compare three different options for $U(\Phi)$, respectively $U(\Phi)=0, U(\Phi)=\lambda^{2} \Phi / 2$ and 
$U(\Phi)=-\lambda^{2} \Phi / 2$, where $\lambda>0$ and real. Application of the Principle of Action as embodied in the Lagrange-Euler equation yields a differential equation for the spatial behavior of the field's potential energy. The homogenous equations are respectively
(a) $\frac{1}{r} \frac{\mathrm{d}^{2}}{\mathrm{~d} r^{2}}(r \Phi)=0$;
(b) $\frac{1}{r} \frac{\mathrm{d}^{2}}{\mathrm{~d} r^{2}}(r \Phi)-\lambda^{2} \Phi=0$ and
(c) $\frac{1}{r} \frac{\mathrm{d}^{2}}{\mathrm{~d} r^{2}}(r \Phi)+\lambda^{2} \Phi=0$

The non-trivial solutions for the first and second case are

$\Phi=\frac{\Phi_{0}}{\lambda r}$ and $\Phi=\Phi_{0} \frac{\exp (-\lambda r)}{\lambda r}$

The first case applies to electromagnetism (for $\Phi_{0}=Q \lambda / 4 \pi \varepsilon_{0}$ ) and Newtonian gravity (for $\Phi_{0}=M G \lambda$ ). The second case applies to Proca's generalization of the Maxwellian field. It reduces to the first case if $\lambda \rightarrow 0$, while keeping $\Phi_{0} / \lambda$ constant. Generically, it represents a field with a format that corresponds with the potential as proposed by Yukawa to explain the short range of the nuclear force. It has been used by the author of this article for the purpose to express the Gravitational Constant in quantum mechanical quantities[3].

Let us now consider the third case. It can be readily verified from (50c) that a non-trivial solution for this case is,

$\Phi=\Phi_{0} \frac{\cos \lambda r+\sin \lambda r}{\lambda r}$

In accordance with the concepts of classical field theory, the field strength can be established as the spatial derivative of the potential $\Phi$. Identifying $\Phi_{0} / \lambda$ as $M G$ and $\lambda$ as a range parameter, we may identify this field strength as a cosmological gravity force $F_{C G}$. Let us compare this force with the Newtonian force $F_{N}$. To do so more explicitly, we compare $F_{N} r^{2}$ with $F_{C G} r^{2}$. The comparison is shown in figure 7.

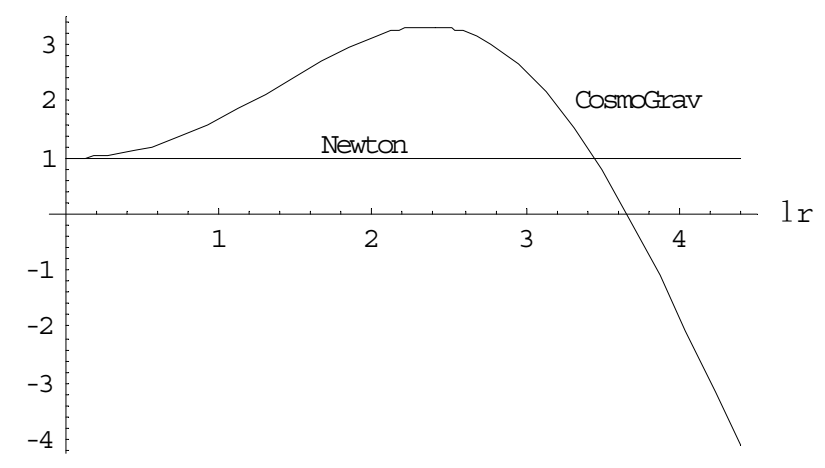

Figure 7: The cosmological gravity force compared with the Newtionian force

This figure shows that, for relative small values of $r$, the cosmological gravity force behaves similarly as the Newtonian force, but that its relative strength over the Newtonian force increases significantly for large values of $r$. This is a similar behavior as heuristically implemented in MOND. The effective range is determined by the parameter $\lambda$. It might 
therefore well be that the cosmological gravity force manifests itself only at cosmological scale. Figure 8 shows that under influence of this force, the rotation curves in the galaxy may assume a flat behavior.

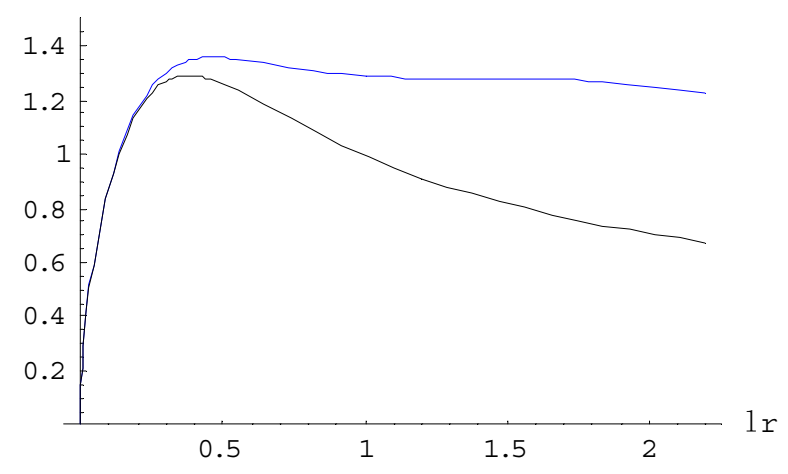

Figure 8: boost of the rotation curve under influence of cosmological gravity.

This hypothetical cosmological gravity shows an intriguing phenomenon. At very far cosmological distance, the attraction of gravity is inverted into repulsion. There is some

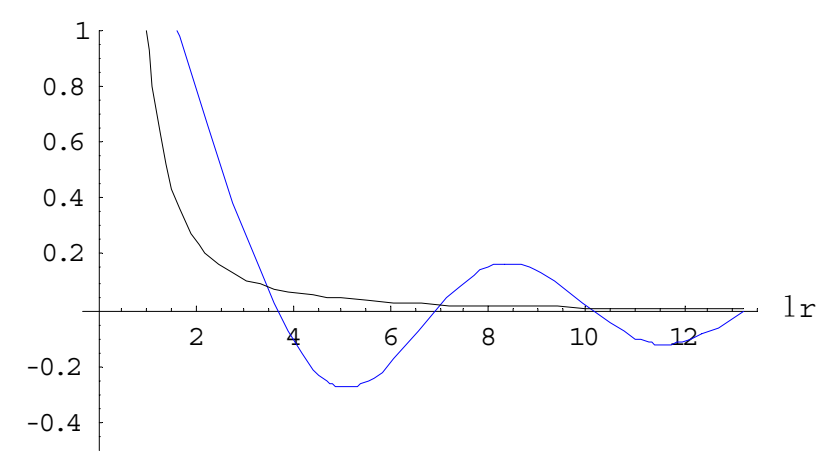

Figure 9: Inversion of the gravity force to antigravity at large cosmological distances. Black: Newtonian. Blue: Cosmological Gravity.

speculation reported in literature that such antigravity is required to explain the phenomenon of dark energy, responsible for the accelerated expansion of the universe [18]. Exploration of this phenomenon is a subject outside the scope of this article. It has to be noted that the solution (49) is not unique. There are more solutions possible by modifying the magnitude of $\sin \lambda r$ over $\cos \lambda r$. I have simply chosen here for the symmetrical solution. Cosmological observations would be required to obtain more insight in this. Such observations are required as well for establishing meaningful values for $\lambda$. In fact, combining a variety of mass density distributions and values for $\lambda$ offer a wealth of galaxy rotation curves that are possible within the validity of the hypothesis.

This view on gravity has an exciting beauty. It unifies the four forces of nature in a single expression for the scalar part of their Lagrangian density, because of

$\odot=-\frac{1}{2} \partial_{\mu} \Phi \partial^{\mu} \Phi+U(\Phi)+\rho \Phi$, where

$U(\Phi)=-\lambda^{2} \Phi / 2$ for gravity, 
$U(\Phi)=\lambda^{2} \Phi / 2$ for the nuclear forces [3,4],

$U(\Phi)=0 \quad$ for electromagnetism

A remaining challenge now is, to harmonize the symmetry that shows up in this classical field view, with Einstein's Field Equation, in which the potential function concept is absent. It might well be that the cosmological parameter $\lambda$, which I have introduced here, can be related with the cosmological constant $\Lambda$ that has been added as a degree of freedom by Einstein in his field equation. Let us see if such could be true. Most generically, Einstein's Field Equation reads as

$$
G_{\mu v}+\Lambda g_{\mu v}=\frac{8 \pi G}{c^{4}} T_{\mu v}
$$

Because no experimental evidence could be found that this constant $\Lambda$ would be different from zero, Einstein regretted its introduction by saying "The introduction of the cosmological constant was the biggest blunder in my life". But I believe, it wasn't. As shown in Appendix B, we may relate Einstein's cosmological constant $\Lambda$ with the cosmological parameter $\lambda$ indeed, as

$$
\Lambda=\lambda^{2}
$$

It is probably fair to say that, apart from the explanation given for dark matter as proposed in this paragraph, this interpretation of the Einstein's cosmological constant, gives a possible support for the cosmological gravity as developed in this article. From textbooks [19], it can be readily concluded that Friedmann's equations predict an accelerated expansion of the universe under a positive value for the cosmological constant. That means that the hypothesis developed in this article does not only give a possible explanation for dark matter, but for dark energy as well.

\section{Comparison with MOND}

It might be instructive to compare this view on cosmological gravity with MOND. MOND is an heuristic approach based on a modification of the gravitational acceleration $g$ such that

$g=\frac{g_{N}}{\mu(x)}$, with $x=g / a_{0}$

where $\mu(x)$ is an interpolation function, $g_{N}\left(=M G / r^{2}\right)$ the Newtonian gravitational acceleration and where $a_{0}$ is an empirical constant velocity. The format of the interpolation function is not known, but the objectives of MOND are met by a simple function [20] like

$\mu(x)=\frac{x}{\sqrt{1+x^{2}}}$.

If $g / a_{0}<<1$, such as happens for large $r,(56)$ reduces to 
$g=\sqrt{a_{0} g_{N}}$

Under this condition, the gravitational acceleration decreases as $r^{-1}$ instead of $r^{-2}$. As a result the orbital velocity curves as a function of $r$ show up as flat curves.

Algebraic evaluation of (57) and (58) results into,

$$
\frac{g}{g_{N}}=\sqrt{\frac{1+\sqrt{1+4 k^{2}(\lambda r)^{4}}}{2}} \quad \text { with } k=\frac{a_{0}}{M G \lambda^{2}}
$$

This expression allows a comparison with the hypothesis as developed in this article. From (52)

$\Phi=\Phi_{0} \frac{\cos \lambda r+\sin \lambda r}{\lambda r} \rightarrow g=-\nabla \Phi=-\frac{\Phi_{0}}{\lambda r^{2}}\left(1+\frac{\lambda^{2} r^{2}}{2}+\ldots.\right)=-\frac{M G}{r^{2}}\left(1+\frac{\lambda^{2} r^{2}}{2}+\ldots.\right)$

hence

$$
\frac{g}{g_{N}}=1+\lambda^{2} r^{2}+\ldots
$$

As illustrated in figure 10, a pretty good fit between (59) and (61) is obtained if

$$
k=\frac{a_{0}}{M G \lambda^{2}}=2.5 \rightarrow a_{0}=2.5 M G \Lambda
$$

Observations on various galaxies have shown that $a_{0}$ can be regarded as a galaxyindependent constant with a value about $a_{0} \approx 1 \times 10^{-10} \mathrm{~m} / \mathrm{s}^{2}$. The implication of (62) is that the cosmological constant $\Lambda$ scales as $M^{-1}$. This is less strange as it might seem, because Einstein's Field Equation with its metric, such as considered in Appendix B, covers the spacetime that emerges from $M$ and not beyond. As I wish to show below, there is no conflict here.

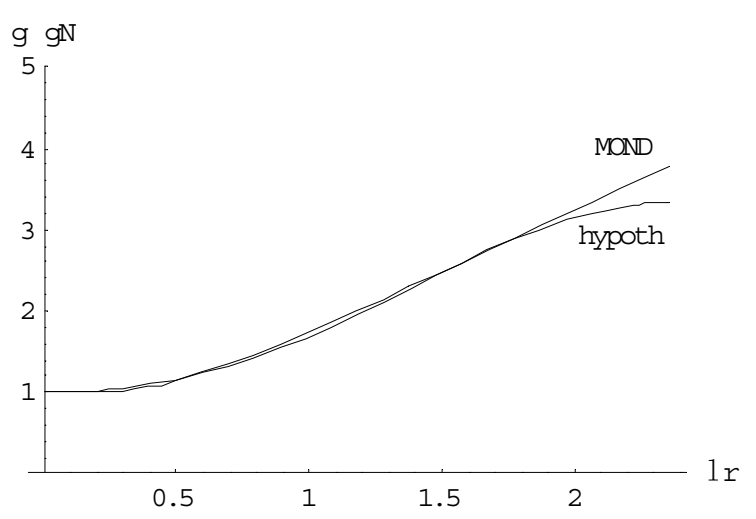

Figure 10: MOND's interpolation function compared with the theory as developed. 
To do so, let us proceed by relating electromagnetism and gravitation by considering the energy density $w$ of the field created by the mass $M$ as

$$
w=\left.\frac{1}{2} \varepsilon_{0}|\nabla \Phi|_{e}\right|^{2} \Leftrightarrow \frac{1}{8 \pi G}|\nabla \Phi|^{2}
$$

I have invoked here $\varepsilon_{G}=(4 \pi G)^{-1}$, in correspondence with the view on gravimagnetism, as outlined before. Relationship (63) allows developing an expression for the energy contained in the mass $M$, under consideration of (60)as

$$
M c^{2}=\int_{V} w \mathrm{~d} V=\frac{1}{8 \pi G}(M G)^{2} \int_{V} f(r) \mathrm{d} V \text { with } f(r)=\left|\frac{\mathrm{d}}{\mathrm{d} r}\left(\frac{\cos \lambda r+\sin \lambda r}{r}\right)\right|^{2} .
$$

This can be evaluated further as

$$
c^{2}=\frac{M G}{8 \pi} \int_{\mathrm{r}_{\infty}}^{\mathrm{r}_{0}} f(r) 4 \pi r^{2} \mathrm{~d} r
$$

In the case that $\lambda=0$, we would have the Newtonian condition. In that case,

$$
\int_{\mathrm{r}_{\infty}}^{\mathrm{r}_{0}} f(r) r^{2} \mathrm{~d} r=\frac{1}{r_{0}} \quad \text { and } \quad M=\frac{2 c^{2} r_{0}}{G}
$$

This result is dimension-wise correct. Like in electromagnetism, there are no means to establish a value for the radius $r_{0}$ of the "pointlike" mass $M$. More interestingly is the case $\lambda>0$. Evaluation of the integral under the condition $\lambda^{2} r_{\infty} \gg>1$ gives

$$
\int_{\mathrm{r}_{\infty}}^{\mathrm{r}_{0}} f(r) r^{2} \mathrm{~d} r=\frac{1}{r_{0}}+\lambda^{2} r_{\infty}
$$

The radius $r_{\infty}$ is the cosmological horizon, which in the Newtonian limit $(\lambda=0)$ does not play any role, while it does in the non-Newtonian case. In fact, the contribution $\lambda^{2} r_{\infty}$ might dominate over the contribution $1 / r_{0}$. It is tempting to relate the cosmological horizon $r_{\infty}$ with Hubble's constant $H_{0}$ and the speed of light as $r_{\infty}=c t_{H}=c / H_{0}$, but actually there is no proof for justification. The practical way for assigning a value to the cosmological horizon is establishing its value from experimental evidence, similarly as is usually done for $r_{0}$. Let us therefore conclude from (64-67)

$$
\lambda^{2}=\Lambda=\frac{c^{2}}{r_{\infty} M G}
$$


The conclusion is that the cosmological constant $\Lambda$ of a galaxy scales as $M^{-1}$ indeed. MOND's acceleration constant (62) can thus be written as

$a_{0}=2.5 \frac{c^{2}}{r_{\infty}}$.

Considering that 1 light year $=9.46 \times 10^{15} \mathrm{~m}$ and that $a_{0} \approx 1 \times 10^{-10} \mathrm{~m} / \mathrm{s}^{2}$, it would make the cosmological horizon $r_{\infty} \approx 2.4 \times 10^{11}$ light year, which seems being quite realistic. It is therefore fair to conclude that the theoretically based hypothesis as presented in this article to explain phenomena that are usually ascribed to dark matter and dark energy, corresponds with the heuristic solution as formulated in Milgrom's MOND.

\section{Synopsis and conclusions}

In this article, the four forces of nature have been unified in a single expression for the scalar part of their underlying Lagrangian density. This Lagrangian density is Proca's generalization of the Maxwellian one. For electromagnetism, Proca's mass term is zero, for the nuclear forces the mass term is positive, for gravity the mass term is negative. As a consequence, the electromagnetic field potential decays as $1 / r$, the nuclear potential decays more aggressively as $\exp (-\lambda r) / r$ and the gravity potential decays less aggressively as $(\cos \lambda r+\sin \lambda r) / r$. Effectively, the gravity potential remains the Newton one in our common world, but is different at cosmological scale. This property explains the cosmological phenomenon that is usually assigned to dark matter. Because of the match in results, the developed model can be regarded as an underlying theory for the heuristic MOND approach, albeit that the prognosis that, at very large cosmological distances, gravity periodically turns on-and-off into antigravity marks a decisive difference. It is proven in this article that the range determining parameter $\lambda$ is related with Einstein's cosmological constant as $\Lambda=\lambda^{2}$. This explains the cosmological phenomenon that our universe is expanding in acceleration, such as predicted by Friedmann's law, under influence of the dark energy that is embodied in the cosmological constant.

This result has been obtained after analyzing the gravity field spread by a rotating massive body from two different perspectives, both with the aim to calculate the force on an orbiting test particle. In the first of these perspectives, an attempt is made to model the gravity field in terms of a naive transposition of Maxwellian laws to gravity, by conceiving currents $\Delta m / \Delta t$ of massive particles as an analogy of electrical currents $\Delta q / \Delta t$. In the second of these perspectives, this force is calculated from the general relativistic metric that describes the space-time of the rotating massive body, thereby trying to give it a gravimagnetic interpretation in terms of modified Maxwellian laws. In this article, the results of both approaches have been compared with the experimental evidence gained from data analysis of measurements from a satellite that from april 2004 till 2014 orbited the earth as exponent of the Gravity B probe experiment, which was initiated in 1961 . This comparison gave rise to some puzzles that might be worthwhile to mention. 
One of these puzzles had to do with the question how the quoted experimental results had to be interpreted. This holds for the geodetic effect as well as for the frame dragging effect, measured by a gyroscope in the satellite orbiting in a circle with a radius of about $7000 \mathrm{~km}$ around the earth's center. The geodetic effect is the same as shown by a planet in our solar system that becomes manifest as a shift of its orbital perihelium. It would therefore be reasonable to expect that the geodetic effect on the gyroscope can be calculated straightforwardly from Mercury's perihelium shift by relating the earth mass with the solar mass and the satellite radius with the radius of Mercury's orbit. The result of this calculations appears being twice the values of those reported in reports on the Gravity $B$ experiment. The second effect, denoted as frame dragging, is caused by the earth's rotation. It is also known as the Lense-Thirring effect, in 1918 straightforwardly calculated by Josef Lense and Hans Thirring from the Einsteinean space-time metric of a massive rotating body. In 1960, this calculation has been redone by Leonard Schiff (Stanford), who projected his analysis on the behavior of a gyroscope in a satellite. Curiously, the Lense-Thirring precession formula $\dot{\Omega}_{L T}$ has the same format as Schiff's formula $\Omega_{L T}$, but is twice in magnitude. I have concluded in this article that the cause of the discrepancy of a factor 2 in both these precession results is due the difference between the actual precession $d \varphi / d t=\dot{\Omega}$ and the gravimagnetic field strength $\Omega_{L T}$, which both have the same dimension $\left[\mathrm{s}^{-1}\right]$. Calculation of the Lense-Thirring effect on the basis of $\dot{\Omega}_{L T}$ gives twice the value reported from the Gravity B probe project. This is consistent with the factor 2 difference in the geodetic effect.

As shown in this article, the Lense-Thirring precession can be calculated by the naive transposition of the Maxwellian laws to gravity. The assignment of a numerical value requires a numerical value for the gravimagnetic constant $\mu_{G}$ next to the gravimagnetic permeability $\varepsilon_{G}=(4 \pi G)^{-1}$. If $\mu_{G} \varepsilon_{G}=c^{-2}$, the result is exactly 4 times smaller than predicted by the (experimentally verified) Lense-Thirring formula for $d \varphi / d t=\dot{\Omega}$. However, if the gravimagnetic field is not radiating, there is no compelling reason why should $c^{2}=c_{G}^{2}$, because, as I have shown, the causality of the gravitational field does not require so. This would make the naïve transposition of the Maxwellian field by maintaining the analogy $\Delta m / \Delta t$ with $\Delta q / \Delta t$ a valid option by assigning an appropriate value to $\mu_{G}$.

This option disappears if credit is given to a gravimagnetic view that represents a full analogy with electromagnetism. This analogy presupposes, next to the identification of a scalar $\Phi=G M / r$ and the vector $\mathbf{A}=(G \mathbf{J} \times \mathbf{x}) / c r^{3}$ in the space-time metric, a coupling between those quantities by the gravitational equivalents of the Faraday induction and the displacement current $(|\mathbf{x}|=r$ and $\mathbf{J}$ is the angular momentum of the central body). This would mean that the gravitational field is radiating, which would not only impose the condition $\mu_{G} \varepsilon_{G}=c^{-2}$, but which also would imply the existence of a spin-1 graviton. The nice feature would be that this view delivers a beautiful analogy between gravimagnetism 
and electromagnetism, albeit that the Lorentz force requires the modification $m \rightarrow 2 m$ and that the relationship between the gravimagnetic field strength $B$ and the particle current density $j$ has to be modified as $B=\mu_{G} j \rightarrow B / 2=\mu_{G} j$.

The beauty of this is no proof. It is probably the better view still to adhere to the gravity field as a spin-2 field, by giving it the following interpretation. It is clear that we have two components, i.e., we have a scalar $\Phi$ and a vectorial $\mathbf{A}$. This gives rise to the equivalents of an electric field and a magnetic field. But there is no proof as yet that these fields are mutually coupled. That would mean that the gravity field is non-radiating. It makes therefore sense, in the author's view, to interpret the gravity field as a spin-2 bosonic field by interpreting a spin- 2 boson as the non-radiating version of a spin-1 boson.

\section{Discussion}

The search for relationships between the four basic forces of nature is an on-going challenge. In this respect, the gravitational force is the most problematic one. Even to the extent that the question if gravity is a basic force indeed, or just emergent from the other basic forces, is still subject to debate. Where electromagnetism and the two nuclear forces are unified in the context of quantum physics, gravity is still on its own. This, in spite of the beauty of Einstein's General Relativity, which has revealed and explained so many gravity related cosmological phenomena. In this article, I have described gravity from a point of view inherited from my earlier work on quantum physics, in which I have positioned quarks as pointlike sources spreading a composite field of energy, identified by me as the Higgs field. It gives rise to bonds of two quarks (mesons) and three quarks (baryons), to which attributes can be assigned that we know as electric charge and mass. In this view, the fields of the strong nuclear force and the weak nuclear force are basic and the fields of electromagnetism and gravity are sourced by the attributes of the quark bonds. In earlier work, this view has enabled me to express the Gravitational Constant in terms of quantum mechanical quantities [3], probably so far considered as just a curiosity. It appeared being possible by combining views of General Relativity with the views of classical field theory. One of the instruments used, was applying Proca's generalization of Maxwell's laws to the nuclear fields of energy. In the context of this article, I have done something similar for gravity. Just by changing the polarity of the mass term in Proca's generalization. In this article, gravity has been modeled as a non-radiating field with a scalar potential next to a vector potential without a two-sided (mutual) coupling in-between. Whether this field can be generalized to a radiating one depends on the question if the gravitational equivalents of the Faraday induction and the displacement current exist in the gravimagnetic view on gravity. If so, my hypothesis could possibly meet serious problems with respect to gauging the field under a gauge invariance constraint of the proposed modified Proca description for the gravity field. It is for that reason I have included Appendix A, in which I have shown that the causality of the gravity field does not need a vectorial bosonic description. 
According to the hypothesis developed in this article, there is no dark matter. There is no dark force either. There is just a generalization of gravity laws. This generalization gives an answer to the anomaly of the rotation curves in galaxies. It also shows how to interpret Einstein's cosmological constant, thereby also giving an explanation for the accelerated expansion of the universe. One of the intriguing consequences is the potential existence of antigravity. This, however, does not mean that there is a domain for it. It is relative. Those who are living in that domain, do not experience antigravity. They just see us living in an antigravity domain instead.

Let me conclude by expressing that my hypothesis is still in a state of immaturity. It gives a challenge, though, for further exploration. The ultimate challenge is proving or disproving it by experiment.

\section{Appendix A: The causality of gravity}

The objective in this appendix is to show how the causality of gravity, such as shown in the wave equation (19) in the main text, is implicitly contained in Einstein's wave equation, even in the case that the source of gravity does not show an amount of rotation energy. This objective implies that we have to solve the weak limit of Einstein's Field Equation for a space-time metric that is given by the line element (17),

$\mathrm{d} s^{2}=g_{00}(r, t) \mathrm{d} q_{0}^{2}+g_{r r}(r, t) \mathrm{d} r^{2}+r^{2} \sin ^{2} \vartheta \mathrm{d} \varphi^{2}+r^{2} \mathrm{~d} \vartheta^{2}$

where $q_{0}=\mathrm{i} c t$.

Note: The space-time $(\mathrm{ict}, r, \vartheta, \varphi)$ is described on the basis of the "Hawking" metric $(+,+,+,+)$.

The weak field limit condition is defined as (18),

$g_{00}(r, t)=1+h_{00}(r, t)$, where $\left|h_{00}(r, t)\right|<<1$, and

$g_{r r}(r, t)=1+h_{r r}(r, t)$, where $\left|h_{r r}(r, t)\right|<<1$.

In empty space, the Einstein Field Equation (16) under this symmetric spherical isotropy, reduces to a simple set of equations for the elements $R_{\mu \mu}$ of the Ricci tensor,

(a): $R_{00}=0 ;(\mathrm{b}): R_{r r}=0 ;(\mathrm{c}): R_{\vartheta}=0$ and (d): $R_{\varphi \varphi}=0$.

The hard task is to calculate these elements. As I wish to show later in Appendix A, the calculation gives the result as shown in Table A1.

(Note: $h^{\prime}$ and $h^{\prime \prime}$ means differentiation, respectively double differentiation of $h$ into $r$; $\dot{h}$ and $\ddot{h}$ means differentiation, respectively double differentiation of $h$ into $t$ )

Adding $(A-3 b)$ to $(A-3 a)$ results in 
$h_{00}^{\prime \prime}-\frac{\ddot{h}_{r r}}{c^{2}}+2 \frac{h_{00}^{\prime}}{r}+2 \frac{h_{r r}^{\prime}}{r}$.

From $(A-3 c)$ and $(A-3 d)$ it follows that

$h_{00}^{\prime}=-h_{r r}^{\prime}$

From $(A-4)$ and $(A-5)$ then follows,

$h_{00}^{\prime \prime}-\frac{\ddot{h}_{00}}{c^{2}}=0 \quad$ and $h_{r r}^{\prime \prime}-\frac{\ddot{h}_{r r}}{c^{2}}=0$,

or written differently,

$\frac{\partial^{2} h_{00}}{\partial r^{2}}-\frac{1}{c^{2}} \frac{\partial^{2} h_{00}}{\partial t^{2}}=0$ and $\frac{\partial^{2} h_{r r}}{\partial r^{2}}-\frac{1}{c^{2}} \frac{\partial^{2} h_{r r}}{\partial t^{2}}=0$, with $\frac{\partial h_{00}}{\partial r}=-\frac{\partial h_{r r}}{\partial r}$.

\section{Table A1: metric tensor and Ricci tensor}

\begin{tabular}{|l|l|}
\hline metric tensor & Ricci tensor \\
\hline$g_{00}=1+h_{00}$ & $R_{00}=-\frac{h_{00}^{\prime}}{2}+\frac{\ddot{h}_{r r}}{c^{2}}+\frac{h_{00}^{\prime}}{4}\left(h_{00}^{\prime}-h_{r r}^{\prime}\right)-\frac{\dot{h}_{r r}}{4}\left(\dot{h}_{00}-\dot{h}_{r r}\right)-\frac{h_{00}^{\prime}}{r}$ \\
\hline$g_{r r}=1+h_{r r}$ & $R_{r r}=-\frac{h_{00}^{\prime}}{2}+\frac{\ddot{h}_{r r}}{c^{2}}-\frac{h_{00}^{\prime}}{4}\left(h_{00}^{\prime}-h_{r r}^{\prime}\right)+\frac{\dot{h}_{r r}}{4}\left(\dot{h}_{00}-\dot{h}_{r r}\right)+\frac{h_{00}^{\prime}}{r}$ \\
\hline$g_{\vartheta \vartheta}=r^{2}$ & $R_{\vartheta \vartheta}=-\frac{r}{2}\left(h_{00}^{\prime}+h_{r r}^{\prime}\right)$ \\
\hline$g_{\varphi \varphi}=r^{2} \sin ^{2}(\vartheta)$ & $R_{\kappa \varphi}=-\frac{r}{2} \sin ^{2}(\vartheta)\left(h_{00}^{\prime}+h_{r r}^{\prime}\right)$ \\
\hline
\end{tabular}

It will be clear that (A-7) is the equation of a wave that propagates in the direction of $r$ with a velocity $c$. This equation is identical in format as Maxwell's wave equation for electromagnetism. It proves the causality of gravity without requiring an equivalent of magnetism in the source of the field.

Calculation of the Ricci tensor

The Ricci tensor is described in expanded form by

$R_{i j}=\sum_{k=0}^{3}\left(\frac{\partial \Gamma_{i j}^{k}}{\partial q_{k}}-\frac{\partial \Gamma_{i k}^{k}}{\partial q_{j}}\right)+\sum_{i=0}^{3} \sum_{k=0}^{3}\left(\Gamma_{i j}^{i} \Gamma_{k i}^{k}-\Gamma_{i k}^{i} \Gamma_{j i}^{k}\right)$.

The Christoffel symbols $\Gamma_{i j}^{k}$ represent functions of the metric elements, such that 
$\Gamma_{i j}^{k}=\frac{1}{2} \sum_{m=0}^{3} g^{k m}\left\{\frac{\partial g_{j m}}{\partial q_{i}}+\frac{\partial g_{i m}}{\partial q_{j}}+\frac{\partial g_{j i}}{\partial q_{m}}\right\}$.

Under symmetric spherical isotropy, only diagonal terms remain, so that the expression reduces to

$R_{i i}=\sum_{k=0}^{3}\left(\frac{\partial \Gamma_{i i}^{k}}{\partial q_{k}}-\frac{\partial \Gamma_{i k}^{k}}{\partial q_{i}}\right)+\sum_{i=0}^{3} \sum_{k=0}^{3}\left(\Gamma_{i i}^{i} \Gamma_{k i}^{k}-\Gamma_{i k}^{i} \Gamma_{i i}^{k}\right)$

and the Christoffel symbols reduce to

$\Gamma_{i j}^{k}=\frac{1}{2 g_{k k}}\left\{\frac{\partial g_{k j}}{\partial q_{i}}+\frac{\partial g_{k i}}{\partial q_{j}}+\frac{\partial g_{j i}}{\partial q_{k}}\right\}$

such that only three different forms remain,

$\Gamma_{k k}^{k}=\frac{1}{2 g_{k k}} \frac{\partial g_{k k}}{\partial q_{k}} ; \quad \Gamma_{i i}^{k}=\frac{1}{2 g_{k k}} \frac{\partial g_{i i}}{\partial q_{k}}(k \neq i)$ and $\Gamma_{i k}^{k}=\frac{1}{2 g_{k k}} \frac{\partial g_{k k}}{\partial q_{i}}$.

Table A2: Christoffel elements and affine connections of the isotropic non-rotating metric

\begin{tabular}{|l|l|l|l|}
\hline$\Gamma_{t t}^{t}$ & $\Gamma_{t r}^{r}$ & & \\
\hline$\Gamma_{r t}^{t}$ & $\Gamma_{r r}^{t}$ & & \\
\hline & & & \\
\hline & & & \\
\hline
\end{tabular}
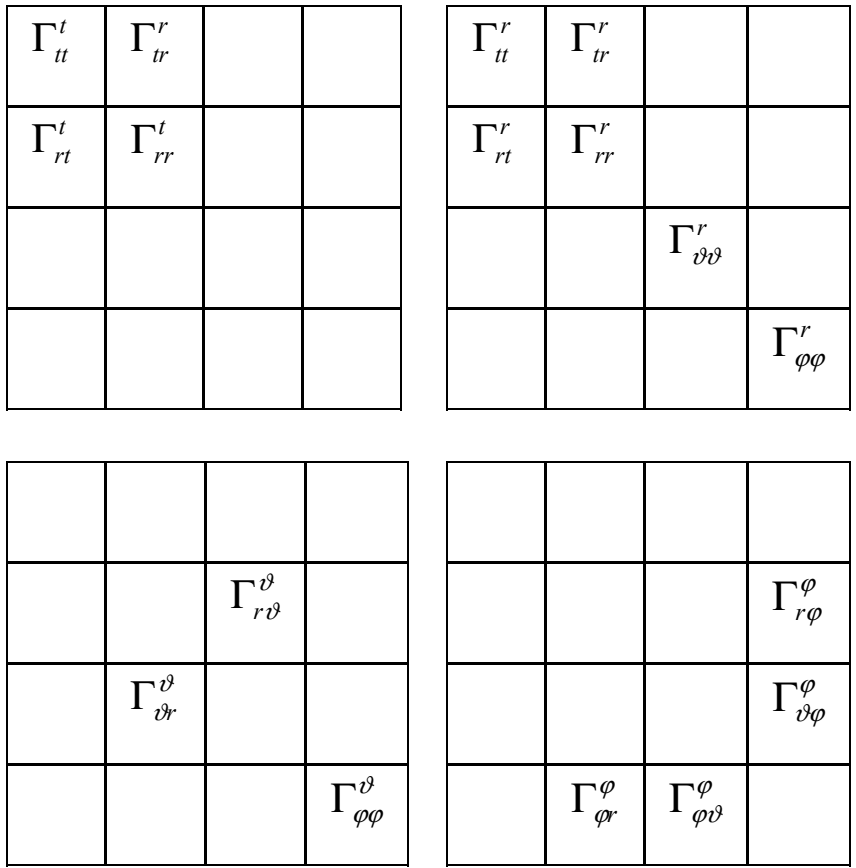

Table A2 shows the Christoffel elements different from zero, where

$$
\begin{array}{lll}
\Gamma_{t t}^{t}=\frac{1}{2 \mathrm{i} c} \dot{h}_{00} & \Gamma_{r r}^{t}=-\frac{1}{2 \mathrm{i} c} \dot{h}_{r r} & \Gamma_{t r}^{t}=\Gamma_{r t}^{t}=\frac{1}{2} h_{00}^{\prime} \\
\Gamma_{t t}^{r}=-\frac{1}{2} h_{00}^{\prime} & \Gamma_{r r}^{r}=\frac{1}{2} h_{r r}^{\prime} & \Gamma_{t r}^{r}=\Gamma_{r t}^{r}=\frac{1}{2 \mathrm{i} c} \dot{h}_{r r}
\end{array}
$$


$\Gamma_{\vartheta \vartheta}^{r}=-\frac{r}{1+h_{r r}} \quad \Gamma_{\varphi \varphi}^{r}=-\frac{r \sin ^{2} \vartheta}{1+h_{r r}}$

$\Gamma_{r \vartheta}^{\vartheta}=\Gamma_{\vartheta r}^{\vartheta}=\frac{1}{r} \quad \Gamma_{\varphi \varphi}^{\vartheta}=-\sin \vartheta \cos \vartheta$

$\Gamma_{r \varphi}^{\varphi}=\Gamma_{\varphi r}^{\varphi}=\frac{1}{r} \quad \Gamma_{\vartheta \varphi}^{\varphi}=\Gamma_{\varphi \vartheta}^{\varphi}=\cot \vartheta$

Application of $(A-13)$ on $(A-10)$ gives the Ricci tensor as listed in Table A1.

Table A2: Christoffel elements and affine connections of the isotropic non-rotating metric
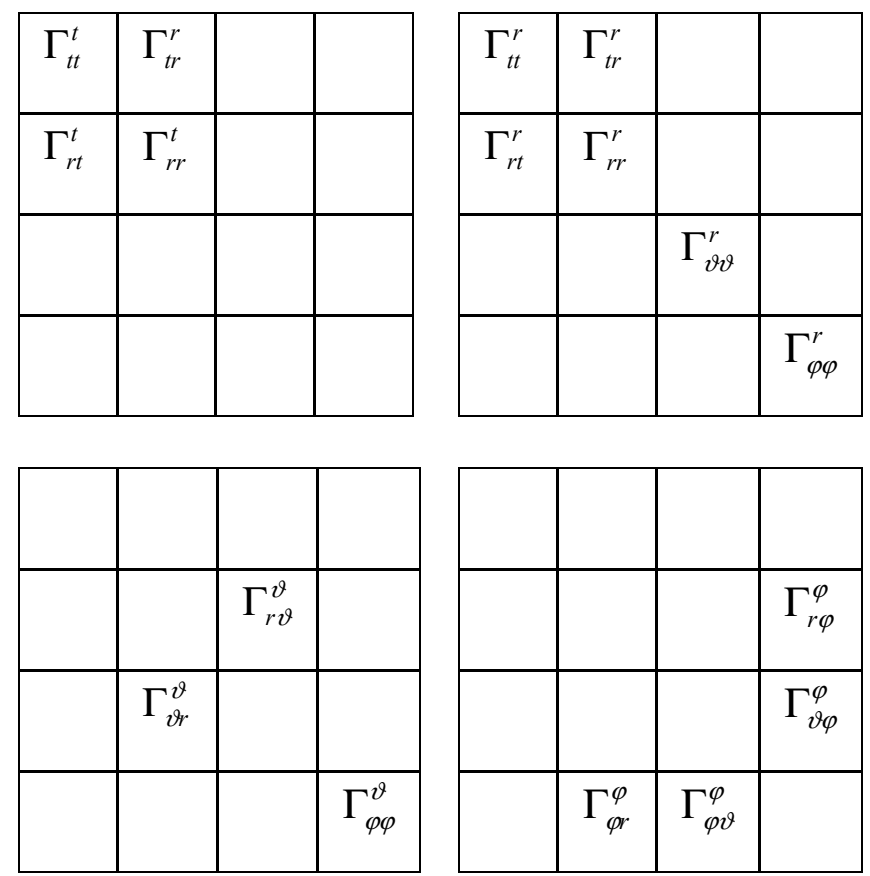

\section{Appendix B: The Cosmological Constant}

So far, the view on cosmological gravity in this article, has been based upon the Lagrangian density concept of a classical field, in which the potential energy $U(\Phi)$ is expressed in terms of a potential function $\Phi$, such that, c.f. (53),

$U(\Phi)=-\lambda^{2} \Phi / 2$

The potential function concept is absent in General Relativity. Instead, the energetic influence of the field is comprised in the elements $g_{\mu \nu}$ of the metric tensor. If we could relate $\Phi$ with the metric tensor, we might possibly relate $\Lambda$ with $\lambda$. Because $\Lambda$ is a constant, we may restrict ourselves to 


$$
G_{00}+\Lambda g_{\mu \nu}=\frac{8 \pi G}{c^{4}} T_{00}
$$

Three equations will be invoked to relate $\Lambda$ with $\lambda$. These are

$$
\begin{aligned}
& \nabla g_{00}=\frac{2}{c^{2}} \nabla \Phi, \\
& G_{00}=\nabla^{2} g_{00}, \\
& \nabla^{2} \Phi+\lambda^{2} \Phi=4 \pi G \frac{T_{00}}{c^{2}} .
\end{aligned}
$$

As I shall show below, the first one is derived from Einstein's geodesic equation of motion. The second is straightforwardly derived from the expression of the Einstein tensor $G_{\mu \nu}$. The third one is derived from the Principle of Action on the Lagrangian density of the field (by applying the Euler-Lagrange equation). Assuming the three equations hold true, elementary algebraic operations on these three equations yield,

$$
G_{00}+\lambda^{2} g_{00}=\frac{8 \pi G}{c^{4}} T_{00}
$$

From (B-2) and (B-4) we find,

$$
\Lambda=\lambda^{2}
$$

The remaining task is to justify the three equations (B-3abc). The methodology to do so can be found in Einstein's article [6,7]. Let us review it briefly. We shall do so for Cartesian spacetimes $\left(t^{\prime}, x, y, z\right)$ and $\left(\tau^{\prime}, x, y, z\right)$, where lab time and proper time are, respectively, normalized as $t^{\prime}=\mathrm{i} c t$ and $\tau^{\prime}=\mathrm{i} c \tau$, where $\mathrm{i}=\sqrt{-1}$, in accordance with a "Hawking metric" $(+,+,+,+)$. The virtue of this metric is the disappearance of the ugly $\eta$ symbols that show up in Minkowski space-time with metric $(-,+,+,+)$. Furthermore, we shall assume that our spacetime is stationary and isotropic.

Equation (B-3a) follows from the 00-component of Einstein's geodesic equation of motion,

$$
\frac{\mathrm{d}^{2} x_{k}}{\mathrm{~d} \tau^{\prime 2}}+\Gamma_{00}^{k}\left(\frac{\mathrm{d} t^{\prime}}{\mathrm{d} \tau^{\prime}}\right)^{2}=0
$$

In the weak field limit of a stationary isotropic field, this equation of motion reduces to

$$
\frac{\mathrm{d}^{2} \mathbf{x}}{\mathrm{d} \tau^{\prime 2}}=\frac{1}{2} \nabla g_{00} \rightarrow \frac{\mathrm{d}^{2} \mathbf{x}}{\mathrm{dt}^{2}}=-\frac{c^{2}}{2} \nabla g_{00}=-\nabla \Phi \rightarrow g_{00}=\frac{2 \Phi}{c^{2}}
$$

This justifies (B-3a). 
Equation (B-3b) follows from the general expression

$$
G_{00}=R_{00}-\frac{1}{2} R
$$

where $R_{00}$ is the 00-component of the Ricci tensor as shown in (A-10) and where $R$ is the Ricci scalar, which is defined as,

$$
R=\sum_{\mu=0}^{3} \sum_{v=0}^{3} g^{\mu v} R_{\mu v} .
$$

In the weak field limit, this reduces in a stationary isotropic field to

$$
R=\sum_{\mu=0}^{3} \sum_{v=0}^{3} \eta^{\mu v} R_{\mu v}=4 R_{00}
$$

where $R_{00}$ can be calculated as

$$
R_{00}=-\nabla^{2} g_{00}
$$

From (B-7) - (B-10), we conclude

$$
G_{00}=\nabla^{2} g_{00}
$$

This justifies (B-3b).

To justify (B-3c), we have to invoke the Principle of Action, by applying the Euler-Lagrange equation to (44). This results into a generalization of Poisson's equation, which now reads as,

$$
\nabla^{2} \Phi+\lambda^{2} \Phi=4 \pi G \rho
$$

where $\rho$ is the spatial mass distribution, which in the stress-energy tensor is identified as

$$
\rho=\frac{T_{00}}{c^{2}}
$$

The justification of (B-3C) follows from (B-12) and (B-13).

\section{References}

[1] M. Milgrom, The Astrophysical Journal, 270, 365 (1983)

[2] E. Verlinde, arXiv:10010785v1 [hep-th] (2010)

[3] E. Roza, Results in Physics, 6, 149 (2016)

[4] E. Roza, Results in Physics, 6, 468 (2016) 
[5] O. Heaviside, The Electrician, 31, 359 (1893)

[6] A. Einstein, Relativity: The Special and General Theory, H. Holt and Company, New York (1916, translation 1920)

[7] T.A. Moore, A General Relativity Workbook, University Science Books, (2013)

[8] https://en.wikipedia.org/wiki/Kerr_metric

[9] B. Masshoon, arXiv: gr-qc/031103v2 (2008)

[10] C.W.F. Everitt et al., Phys.Rev. Lett. 106, 221101 (2011)

[11] J. Mester and the GP-B Collaboration, Séminaire Poincaré IX, 55 (2006)

[12] J. Lense and H. Thirring, Phys. Zeitschrift 19, 156 (1918)

[13] H. Pfister, General Relativity and Gravitation 39 (11), 1735 (2007)

[14] www.math.toronto.edu/ colliand/426 03/Papers03/C Pollock.pdf

[15] www.physicspages.com/2013/04/07/magnetic-field-of-rotating-sphere-of-charge/

[16] L.I. Schiff, Proc. N.A.S., 46, 877 (1960)

[17] L. Iorio, arXiv:gr-qc/0411084 (2004)

[18] L.M. Krauss, Scientific American, 59 (1999)

[19] https://ned.ipac.caltech.edu/level5/Carroll2/Carroll1 2.html

[20] G. Gentile, B. Famae and W.G.J . de Blok, J. Astronomy and Astrophysics, 527, 76 (2011)

(C) 2016 by the author; licensee Preprints, Basel, Switzerland. This article is an open access article distributed under the terms and conditions of the Creative Commons by Attribution (CC-BY) license (http://creativecommons.org/licenses/by/4.0/). 